\title{
Organic Molecules with Inverted Gaps between First Excited Singlet and Triplet States and Appreciable Fluorescence Rates
}

Robert Pollice, ${ }^{1,2}$ Pascal Friederich, ${ }^{1,2,3}$ Cyrille Lavigne, ${ }^{1,2}$ Gabriel dos Passos Gomes, ${ }^{1,2}$ Alán AspuruGuzik $*, 1,2,4,5$

${ }^{1}$ Chemical Physics Theory Group, Department of Chemistry, University of Toronto, 80 St George St, Toronto, Ontario M5S 3H6, Canada.

${ }^{2}$ Department of Computer Science, University of Toronto, 214 College St., Toronto, Ontario M5T 3A1, Canada.

${ }^{3}$ Institute of Nanotechnology, Karlsruhe Institute of Technology, Hermann-von-Helmholtz-Platz 1 , 76344 Eggenstein-Leopoldshafen, Germany.

${ }^{4}$ Vector Institute for Artificial Intelligence, 661 University Ave Suite 710, Toronto, Ontario M5G 1M1, Canada.

${ }^{5}$ Lebovic Fellow, Canadian Institute for Advanced Research (CIFAR), 661 University Ave, Toronto, Ontario M5G 1M1, Canada.

*Corresponding author: aspuru@utoronto.ca

\section{Abstract}

One of the recent proposals for the design of state-of-the-art emissive materials for organic light emitting diodes (OLEDs) is the principle of thermally activated delayed fluorescence (TADF). The underlying idea is to enable facile thermal upconversion of excited state triplets, which are generated upon electron-hole recombination, to excited state singlets by minimizing the corresponding energy difference resulting in devices with up to $100 \%$ internal quantum efficiencies (IQEs). Ideal emissive materials potentially surpassing TADF emitters should have both negative singlet-triplet gaps and appreciable fluorescence rates to maximize reverse intersystem crossing (rISC) rates from excited triplets to singlets while minimizing ISC rates and triplet state occupation leading to long-term operational stability. However, molecules with negative singlet-triplet gaps are extremely rare and, to the best of our knowledge, not emissive. In this work, based on computational studies, we describe the first molecules with negative singlet-triplet gaps and considerable fluorescence rates and show that they are more common than hypothesized previously.

\section{Introduction}

Hund's first rule ${ }^{1}$ predicts that the first excited state of closed-shell molecules is a triplet state of lower energy than the first excited singlet state. This prediction holds for all but a handful of all known compounds, both organic and inorganic. ${ }^{2,3}$ Hence, it is the basis for Jablonski diagrams ${ }^{4}$ in educational material about electronic spectra of molecules illustrating that it is almost considered a basic truth in chemistry. ${ }^{5-12}$ Molecules violating Hund's first rule in their first excited singlet and triplet energies are said to possess an "inverted" singlet-triplet gap (herein termed the INVEST property). Very few, i.e. on the order of 10 , organic INVEST molecules were predicted on the basis of computations alone $e^{2,13,14}$ with little to no experimental evidence, ${ }^{15,16}$ and, as far as we know, no inorganic INVEST molecule is known to date. Besides inherently inverted molecules, it has been shown in recent years that the influence of the environment can also make the gap negative, ${ }^{17}$ for instance in exciplexes, ${ }^{18}$ through strong light-matter coupling in microcavities, ${ }^{19}$ and polarizable environments. ${ }^{20}$ Moreover, the possibility of inverted singlet-triplet gaps but in higher excited states was also investigated. ${ }^{21}$ 
Nevertheless, two recent publications appeared almost simultaneously, sparking new interest in INVEST molecules and their potential applications in photocatalysis, and in organic light-emitting diodes (OLEDs). ${ }^{22,23}$ The two molecules reported were both based on phenalene ${ }^{24}$ with a distinct degree of nitrogen substitution. In these structures, the inverted gaps emerge as a result of both very small exchange integrals, due to minimal spatial overlap between the HOMO and the LUMO, and significant double excitation character in the electronic transitions leading to stabilization of the first excited singlet relative to the first excited triplet via spin polarization. This is important, as it was shown previously that inherent single-excitation methods, including time-dependent density functional approximations (TD-DFAs) with GGA, meta-GGA and hybrid functionals, are unable to describe singlettriplet inversion. ${ }^{22,23}$

Importantly, both reported INVEST molecules have symmetry-forbidden $\mathrm{S}_{1}-\mathrm{S}_{0}$ transitions and are likely very poor emitters. Provided INVEST molecules with appreciable fluorescence rates were found, they could have the potential to become the next generation of OLED materials ${ }^{17,25}$ because of fast reverse intersystem crossing (i.e., TADF without activation), high emission rates, and a thermodynamic equilibrium that disfavors triplets, and, hence, minimizes triplet nonradiative decay processes that shorten device lifetimes. ${ }^{17}$ Based on computational evidence, we herein reveal many novel organic INVEST molecules with appreciable fluorescence rates. Overall, we observe that the singlet-triplet gap, the fluorescence rates, and the absorption wavelength can be tuned by modification, especially nitrogen substitution, of the phenalene core. We also observe that substitution with electron-donating and electron-withdrawing groups, via the "push-pull" effect,, ${ }^{26,27}$ can lead to azaphenalenes with increased oscillator strengths, hence also to increased fluorescence rates, while maintaining inverted singlet-triplet gaps. Finally, we observe that systematic optimization of substituted azaphenalenes for high oscillator strength, small singlet-triplet gap, and absorption wavelength leads to a rich chemical space of highly emissive INVEST molecules covering essentially the entire visible light spectrum.

\section{Computational Details}

Ground state conformational ensembles were generated using crest $^{28}$ (version 2.10.1) with the iMTD$\mathrm{GC}^{29,30}$ workflow (default option) at the GFNO-xTB ${ }^{31}$ level of theory. The lowest energy conformers were first reoptimized using $\mathrm{xtb}^{32}$ (version 6.3.0) at the GFN2-xTB ${ }^{33,34}$ level of theory, followed by another reoptimization using Orca ${ }^{35,36}$ (version 4.2.1) at the B3LYP ${ }^{37-39} /$ cc-pVDZ $^{40}$ level of theory. The corresponding geometries were used for subsequent ground and excited state single-point calculations. Single points at the $\omega B 2$ PLYP $^{41} /$ def2-SVP, ${ }^{42}$ and DLPNO-NEVPT2 $(6,6)^{43} /$ def2-SV $(P)^{42}$ levels of theory were performed using $\operatorname{Orca}^{35,36}$ (version 4.2.1), single points at the $A D C(2)^{44-50} / c c-p V D Z,{ }^{40}$ $A D C(3)^{44-50} / \mathrm{cc}-\mathrm{pVDZ},{ }^{40}$ EOM-CCSD ${ }^{51-55} / \mathrm{cc}-\mathrm{pVDZ},{ }^{40}$ FNO-EOM-CCSD ${ }^{51-59} / \mathrm{cc}-\mathrm{pVDZ}^{40}$ with $98.85 \%$ of the total natural population, and SA-SF-PBE50 ${ }^{60-65} /$ def2-SVP $^{40}$ levels of theory were performed using Q$\mathrm{Chem}^{66}$ (version 5.2). Ground and excited geometry optimizations for adiabatic state energy differences at the $\omega B 2$ PLYP $^{41} /$ def2-SV $(P)^{42}$ level of theory were performed in Orca $^{35,36}$ (version 4.2.1) using numerical gradients. For all excited state single point calculations, four roots were chosen each for both the singlet and the triplet manifold. For the ground and excited state geometry optimizations, two roots were chosen each. Fluorescence rate estimates are based on absorption oscillator strengths, and converted based on well-established equations from the literature. ${ }^{67}$ 


\section{Results}

Benchmarking. We set out to find efficient methods able to predict the singlet-triplet inversion, which are suitable for high-throughput virtual screening. Several efficient approaches were compared against benchmark methods for molecules 1 and $\mathbf{2}$ (Scheme 1). Table 1 shows the results of several computational excited state techniques of varying computational cost including two particularly efficient families of methods that include double excitations, namely double-hybrid TD-DFAs ${ }^{68-71}$ $\left(\omega B 2\right.$ PLYP $\left.^{41}\right)$ and spin-flip TD-DFAs ${ }^{60,61}$ (SA-SF-PBE50 ${ }^{60-65}$ ). Using $\omega B 2 P L Y P$, we could estimate vibrational contributions to the singlet-triplet gap by performing excited singlet and triplet geometry optimizations. Due to their rigid structures, the energy difference between singlet and triplet minima (also termed adiabatic gap) is almost identical to the singlet-triplet gap at the Franck-Condon point (also termed vertical gap) for both $\mathbf{1}$ and $\mathbf{2}$. Hence, we decided to continue our study using the latter as an approximation to the gap between minima. We note that $\omega$ B2PLYP only reproduces an inverted singlet-triplet gap for $\mathbf{2}$, but not for $\mathbf{1}$. This is the result of a systematic and correctable offset compared to benchmark methods like ADC(2) or EOM-CCSD (vide infra).<smiles>C1=CC=Cc2ccccc2C=1</smiles>

1<smiles>C1=NC2=NC=NC3=NC=NC(=N1)N23</smiles>

2

Scheme 1. Structures of azaphenalenes used for initial benchmarking of singlet-triplet gaps.

Table 1. Benchmarking of excited-state energy differences of $\mathbf{1}$ and $\mathbf{2}$. Both double-hybrid TD-DFAs and spin-flip TD-DFAs can reproduce inverted gaps.

\begin{tabular}{|c|c|c|c|c|}
\hline \multirow{2}{*}{ Method } & \multicolumn{2}{|c|}{1} & \multicolumn{2}{|c|}{2} \\
\hline & $\Delta \mathrm{E}\left(\mathrm{S}_{0}-\mathrm{S}_{1}\right)[\mathrm{eV}]$ & $\Delta \mathrm{E}\left(\mathrm{S}_{1}-\mathrm{T}_{1}\right)[\mathrm{eV}]$ & $\Delta \mathrm{E}\left(\mathrm{S}_{0}-\mathrm{S}_{1}\right)[\mathrm{eV}]$ & $\Delta \mathrm{E}\left(\mathrm{S}_{1}-\mathrm{T}_{1}\right)[\mathrm{eV}]$ \\
\hline$A D C(3) / c c-p V D Z$ & 0.777 & -0.092 & 2.665 & -0.109 \\
\hline$A D C(2) / c c-p V D Z$ & 1.038 & -0.160 & 2.578 & -0.278 \\
\hline EOM-CCSD/cc-pVDZ & 1.092 & -0.099 & 2.791 & -0.180 \\
\hline FNO-EOM-CCSD/cc-pVDZ & 1.126 & -0.104 & 3.418 & -0.214 \\
\hline DLPNO-NEVPT2(6,6)/def2-SV(P) & 1.112 & -0.189 & 2.552 & -0.344 \\
\hline$\omega B 2 P L Y P / d e f 2-S V P$ & 1.316 & 0.042 & 3.028 & -0.218 \\
\hline$\omega B 2 P L Y P /$ def2-SV(P) (vertical) & 1.347 & 0.046 & 3.089 & -0.198 \\
\hline$\omega B 2 P L Y P / d e f 2-S V(P)$ (adiabatic) & 1.296 & 0.055 & 3.045 & -0.188 \\
\hline SA-SF-PBE50/def2-SVP & 1.095 & -0.109 & 2.909 & -0.181 \\
\hline
\end{tabular}

Effect of Core Structure. Compounds $\mathbf{1}$ and $\mathbf{2}$ are isoelectronic and differ only by substitution of $\mathrm{C}-\mathrm{H}$ with $\mathrm{N}$. Hence, we decided to explore all structures resulting from systematic permutations of such nitrogen substitutions (Scheme 2). 
<smiles></smiles>

\section{$\mathrm{A}=\mathrm{C}-\mathrm{H}$ or $\mathbf{N}$}

Scheme 2. Systematic substitution of $\mathrm{C}-\mathrm{H}$ with $\mathrm{N}$ in azaphenalenes cores.

Figure 1 illustrates the predicted properties of the resulting compounds, at the EOM-CCSD/cc-pVDZ level of theory, with the singlet-triplet gap on the abscissa and the oscillator strength for the $\mathrm{S}_{0}-\mathrm{S}_{1}$ transition $\left(f_{12}\right)$ on the ordinate. It shows that there are several INVEST molecules with non-zero oscillator strength. From these molecules, we selected four, marked in red in Figure 1 and depicted in Scheme 3, because of their favorable trade-off between the singlet-triplet gap and the oscillator strength, their distinct excitation energies and because synthetic procedures for compounds with these core structures have been reported. ${ }^{72-88}$ State energy differences, oscillator strengths and estimated fluorescence rate constants of 1-6 are summarized in Table 2.

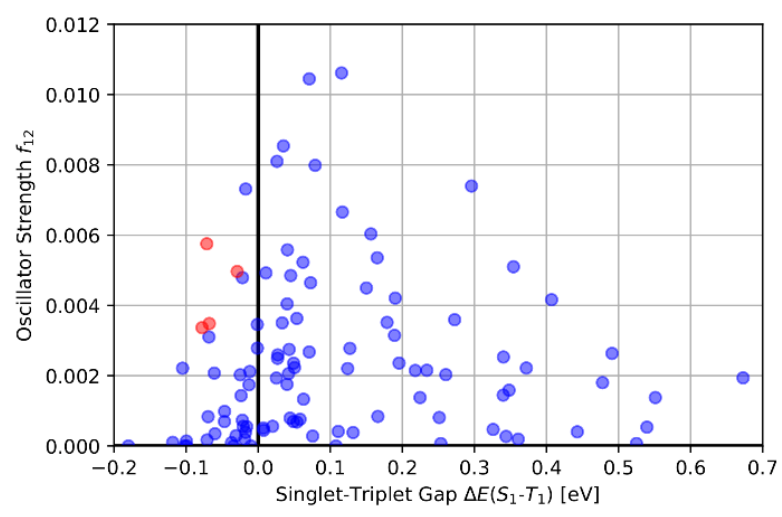

Figure 1. Map of singlet-triplet gaps and oscillator strengths of all permutations of substituting $\mathrm{C}-\mathrm{H}$ with $\mathrm{N}$ in azaphenalenes at the EOM-CCSD/cc-pVDZ level of theory. Red data points show structures selected for further investigations.<smiles></smiles>

3<smiles>C1=CC2=CC=NC3=NC=NC(=C1)N23</smiles>

4<smiles>C1=CC2=NC=NC3=NC=NC(=C1)N23</smiles>

5<smiles>C1=NC2=CC=NC3=NC=NC(=C1)N23</smiles>

6

Scheme 3. Azaphenalenes with the best trade-off between the singlet-triplet gap and the fluorescence rate. 
Table 2. Excited-state energy differences and oscillator strengths of the $\mathrm{S}_{0}-\mathrm{S}_{1}$ transition, and estimated fluorescence rates of the $S_{1}-S_{0}$ transition for compounds 1-6 at the EOM-CCSD/cc-pVDZ level of theory.

\begin{tabular}{ccccc}
\hline $\begin{array}{c}\text { EOM-CCSD/cc- } \\
\text { pVDZ }\end{array}$ & $\Delta \mathrm{E}\left(\mathrm{S}_{0}-\mathrm{S}_{1}\right)[\mathrm{eV}]$ & $\Delta \mathrm{E}\left(\mathrm{S}_{1}-\mathrm{T}_{1}\right)[\mathrm{eV}]$ & $\begin{array}{c}\text { Oscillator strength } \\
\mathrm{f}_{12}\end{array}$ & $\begin{array}{c}\text { Fluorescence } \\
\text { rate } \mathrm{k}_{\mathrm{F}}\left[10^{6} \mathrm{~s}^{-1}\right]\end{array}$ \\
\hline $\mathbf{1}$ & 1.092 & -0.099 & 0.000 & 0.00192 \\
$\mathbf{2}$ & 2.791 & -0.180 & 0.000 & 0.0105 \\
$\mathbf{3}$ & 1.659 & -0.068 & 0.003 & 0.416 \\
$\mathbf{4}$ & 2.012 & -0.029 & 0.005 & 0.872 \\
$\mathbf{5}$ & 2.251 & -0.078 & 0.003 & 0.741 \\
$\mathbf{6}$ & 2.209 & -0.071 & 0.006 & 1.22 \\
\hline
\end{tabular}

Effect of Substitution. Although several new molecules with inverted gaps were obtained, none were optically bright. Hence, we decided to look at the impact of both electron-donating and electronwithdrawing substituents on the properties, as well as probing both mesomeric and inductive effects. Hence, we selected a set of 18 substituents that are both common and small and computed the properties for all distinct monosubstituted analogues of compounds 1-6, as depicted in Scheme 4 . The corresponding property map, at the EOM-CCSD/cc-pVDZ level of theory, is shown in Figure 2. A single substituent can change the singlet-triplet gap by several tenths of electronvolts and the oscillator strength can be increased up to one order of magnitude. In this small set of monosubstituted molecules, there are already a few INVEST molecules with appreciable oscillator strength. These observations suggest that both the singlet-triplet gap and the oscillator strength can be tuned to a significant extent by substituents and that systematic optimization of both these properties is feasible.

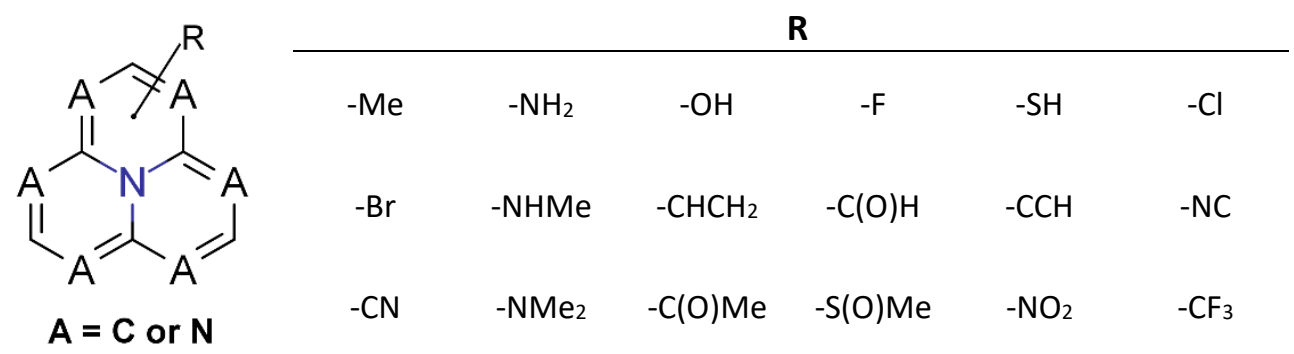

Scheme 4. Systematic monosubstitution of compounds 1-6 with diverse substituents.

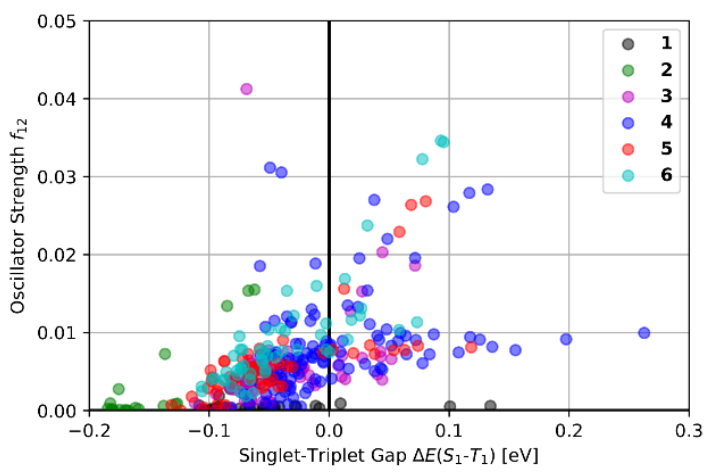

Figure 2. Map of singlet-triplet gaps and oscillator strengths of systematic monosubstituted compounds 1-6 at the EOM-CCSD/cc-pVDZ level of theory.

Optimization of Oscillator Strength. To start optimizing oscillator strength while keeping the singlettriplet gap negative, we needed to establish a robust computational protocol that predicts trends in the INVEST property, as well as the oscillator strength, and can be efficiently applied to larger 
molecules. Hence, all EOM-CCSD/cc-pVDZ results, both singlet-triplet gaps and oscillator strengths, of the core structures and monosubstituted compounds were compiled as a benchmark dataset. Figure 3 compares this dataset against computationally less expensive methods. It shows that $A D C(2) / c c-$ pVDZ generally shows the closest agreement with EOM-CCSD/cc-pVDZ, but at too high a cost for screening as we only saw a timing reduction of roughly $10 \%$. $\omega B 2 P L Y P / d e f 2-S V P$ offers the best tradeoff between cost and accuracy. We observed a more than 200-fold timing reduction compared to EOMCCSD/cc-pVDZ, while still faithfully reproducing trends in both singlet-triplet gaps and oscillator strengths.

a)

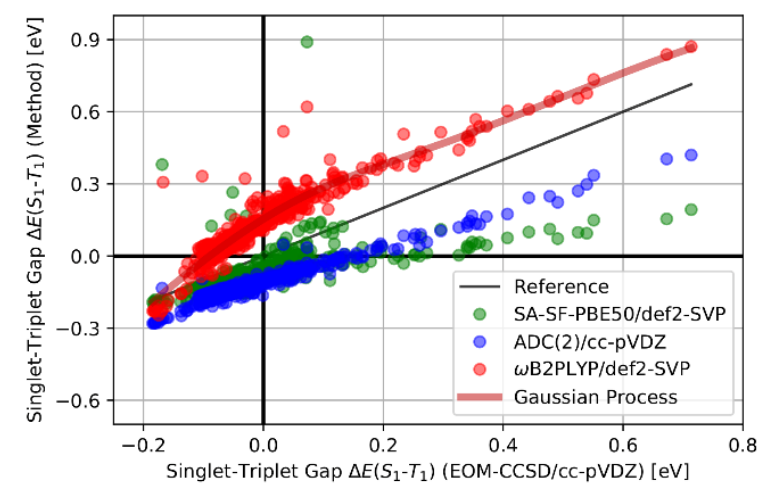

b)

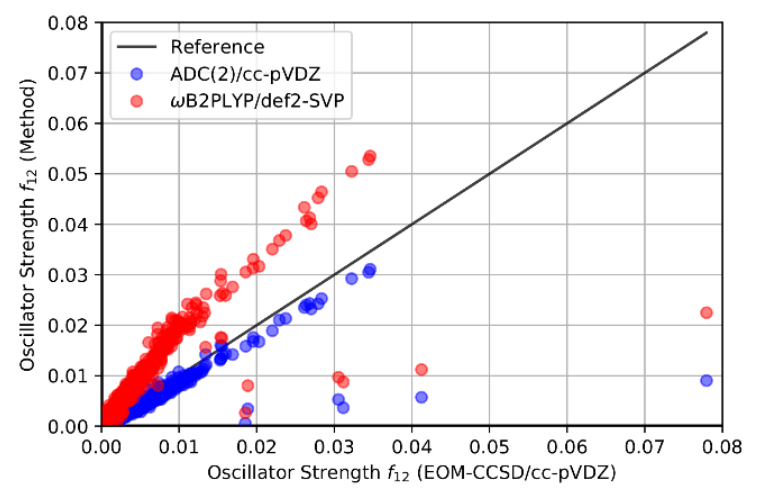

Figure 3. Benchmarking of computational methods for singlet-triplet gaps (a) and oscillator strengths (b). WB2PLYP/def2-SVP offers the best trade-off between cost and accuracy. The calculation of oscillator strengths was not available for SA-SF-PBE50/def2-SVP.

To correct for the systematic shift in the $\omega B 2 P L Y P / d e f 2-S V P$ singlet-triplet gaps, we estimated the offset against an EOM-CCSD/cc-pVDZ calculation using Gaussian process regression. The estimated offset is $0.15 \pm 0.05 \mathrm{eV}$ at a singlet-triplet gap of $0 \mathrm{eV}$. Hence, molecules were optimized by keeping the $\omega B 2 P L Y P / d e f 2-S V P$ singlet-triplet gap below $0.15 \mathrm{eV}$, while maximizing the oscillator strength simultaneously. We note that outliers in the oscillator strength diagrams (cf. Figure $3 \mathrm{~b}$ ) are likely errors from EOM-CCSD/cc-pVDZ as correlating the ADC(2)/cc-pVDZ and $\omega B 2 P L Y P / d e f 2-S V P$ results does not show considerable outliers. In addition, to correct for systematic discrepancies in the computed vertical $S_{1}$ excitation energies and estimate the solvatochromic shift of the studied compounds in solution, we compiled experimental UV-VIS absorption data in solution from the literature and used linear regression for correction. All predicted absorption wavelengths provided are corrected that way. The underlying data is found in the Supporting Information.

Consequently, INVEST molecules were optimized by systematic structural modification and fine-tuning of properties. The corresponding progress is depicted in Figure 4. Importantly, the abscissa, indicating the molecule number, essentially illustrates how we explored the chemical space over time. Some important structures along the optimization are marked with diamond markers in Figure 4a, with red markers in Figure $4 \mathrm{~b}$, and highlighted in Table 3 . These results demonstrate that INVEST molecules with appreciable fluorescence rates can indeed be designed and are likely not as rare as previously hypothesized. ${ }^{22}$ 
a)

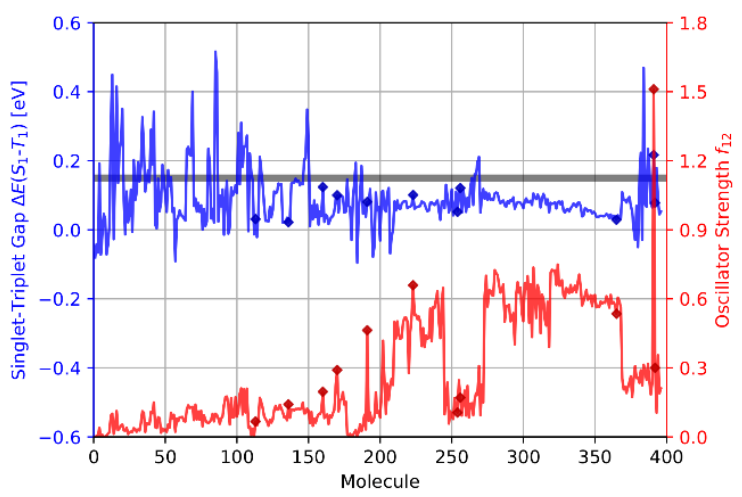

b)

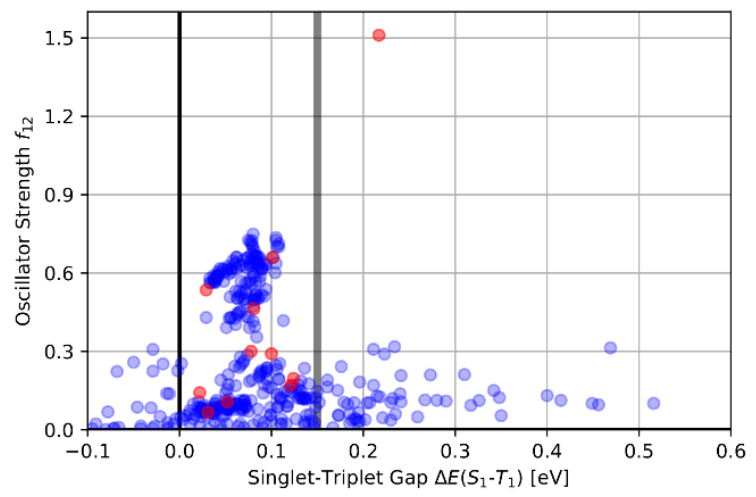

Figure 4. Optimization progress for both the singlet-triplet gap and oscillator strength (a) and property map (b) of all compounds investigated during the optimization. Significant structures are marked with diamond markers (a) and red markers (b), respectively.

Table 3. Important structures along the optimization trajectory, aimed at INVEST molecules with appreciable oscillator strength, and their properties. Absorption wavelengths, $\lambda\left(S_{0}-S_{1}\right)$, are corrected based on experimental data (vide supra, details in the Supporting Information).

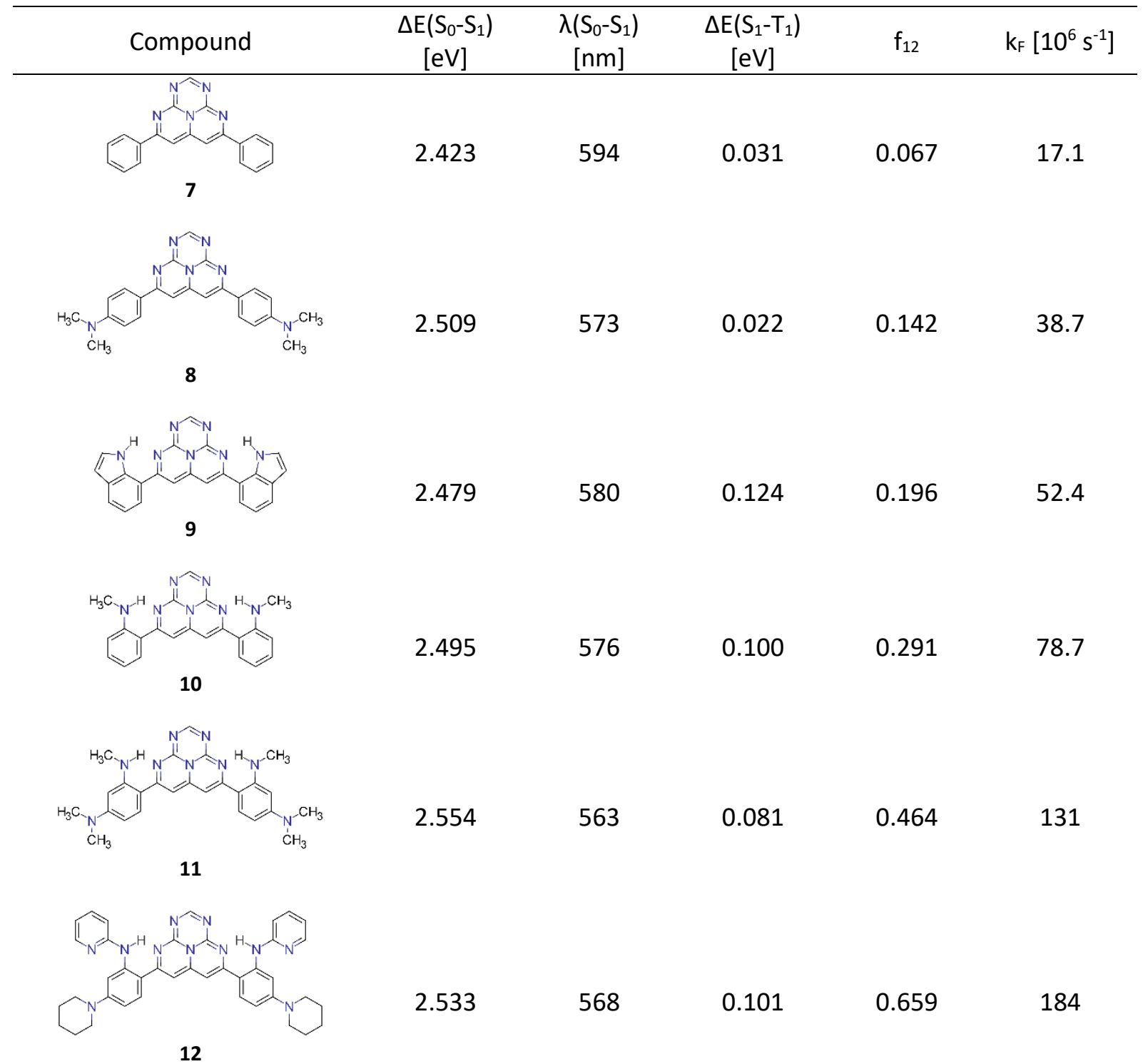




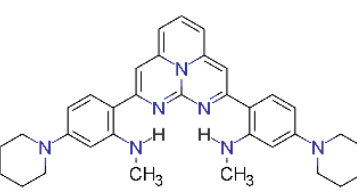

13

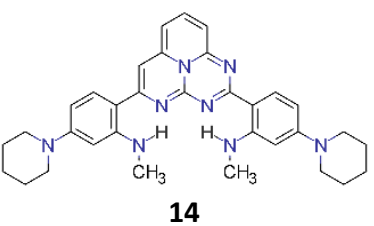

2.345

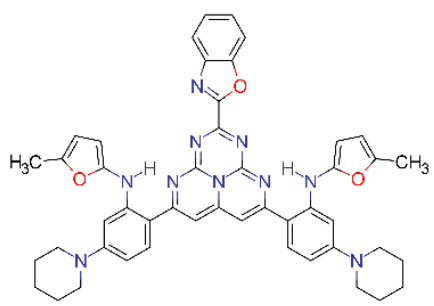

15

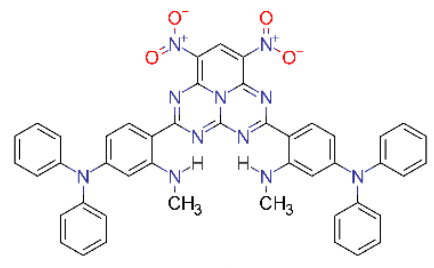

16

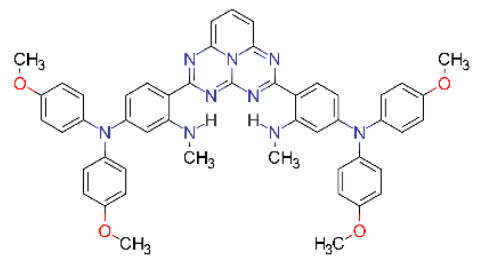

17

Discovery and Optimization of Blue Emitters. The previous optimization turned out no potential blue emitters, a color of particular importance in optoelectronic applications. ${ }^{25,89-97}$ Before carrying out a more focused investigation towards INVEST molecules with appreciable fluorescence rates, we tested a few modifications of molecules $\mathbf{1}$ and $\mathbf{2}$ to find out what structural features undo the inversion, making the singlet-triplet gap positive. One change that did not undo it, but also blue-shifted the excitation significantly is the modification leading to azacyclopenta[cd]phenalene ${ }^{98} 18$, shown in Scheme 5a. Hence, analogously to before, we explored all structures resulting from systematic permutations of all possible substitutions of $\mathrm{C}-\mathrm{H}$ with $\mathrm{N}$ (Scheme $5 \mathrm{~b}$ ). 
a)<smiles></smiles>

18 b)

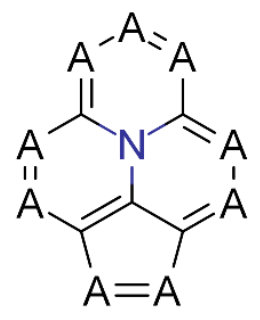

$\mathbf{A}=\mathbf{C}-\mathbf{H}$ or $\mathbf{N}$

Scheme 5. Structure of azacyclopenta[cd]phenalene $\mathbf{1 8}$ (a) and systematic substitution of C-H with N in azacyclopenta[cd]phenalene cores (b).

Figure 5a shows the map of the singlet-triplet gaps and the oscillator strengths at the EOM-CCSD/ccpVDZ level of theory and Figure $5 \mathrm{~b}$ shows the map of the singlet-triplet gaps and the vertical excitation energies. Compared to Figure 1, the lowest singlet-triplet gaps are larger, the range of singlet-triplet gaps is narrower, and the range of oscillator strengths is wider. We identified four core structures that showed the best trade-off between singlet-triplet gap, oscillator strength and vertical excitation energy. Their structures are depicted in Scheme 6 and their properties are summarized in Table 4. Compounds 20-22 are derivatives of $\mathbf{4}$ and $\mathbf{6}$, some of the most promising INVEST core structures identified in the previous sections, thus it was not very surprising these structures would be among the ones with the best combination of properties for blue INVEST emitters. Notably, none of the four azacyclopenta[ $[c d]$ phenalenes $\mathbf{1 9 - 2 2}$ have been reported in the literature before, and only derivatives of $\mathbf{1 8}$ have been synthesized previously. ${ }^{99-101}$

a)

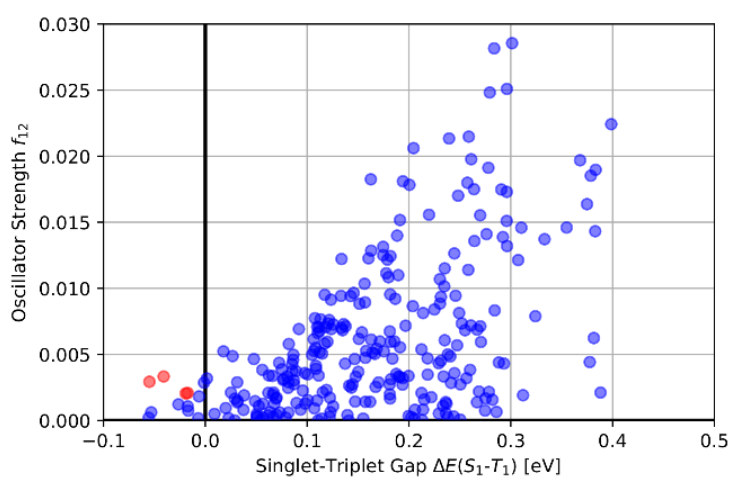

b)

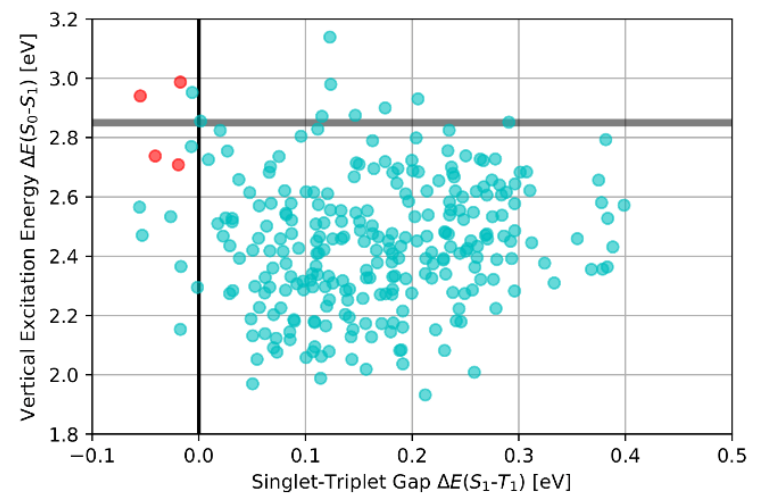

Figure 5. Maps of singlet-triplet gaps, oscillator strengths (a) and vertical excitation energies (b) of all permutations of substituting $\mathrm{C}-\mathrm{H}$ with $\mathrm{N}$ in azacyclopenta[cd]phenalenes at the EOM-CCSD/cc-pVDZ level of theory. Red data points show structures with a good trade-off between the singlet-triplet gap, oscillator strength, and vertical excitation energy. The horizontal gray line in (b) indicates a vertical excitation energy of $2.85 \mathrm{eV}$ corresponding to about $468 \mathrm{~nm}$, after correcting for the solvatochromic shift. 


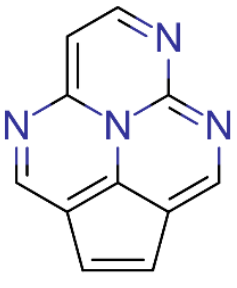

19<smiles>c1nc2ccc3ccc4cnc(n1)n2c34</smiles>

20<smiles>c1nc2ncc3ccc4cnc(n1)n2c34</smiles>

21<smiles>c1nc2cnc3ncc4c-3c(n1)ncc4=N2</smiles>

22

Scheme 6. Azacyclopenta[cd]phenalenes with the best trade-off between singlet-triplet gap, oscillator strength, and vertical excitation energies.

Table 4. Excited state energy differences and oscillator strengths of the $\mathrm{S}_{0}-\mathrm{S}_{1}$ transition, and estimated fluorescence rates of the $S_{1}-S_{0}$ transition for compounds 18-22 at the EOM-CCSD/cc-pVDZ level of theory.

\begin{tabular}{cccccc}
\hline Compound & $\Delta \mathrm{E}\left(\mathrm{S}_{0}-\mathrm{S}_{1}\right)[\mathrm{eV}]$ & $\lambda\left(\mathrm{S}_{0}-\mathrm{S}_{1}\right)[\mathrm{nm}]$ & $\Delta \mathrm{E}\left(\mathrm{S}_{1}-\mathrm{T}_{1}\right)[\mathrm{eV}]$ & $\mathrm{f}_{12}$ & $\mathrm{k}_{\mathrm{F}}\left[10^{6} \mathrm{~s}^{-1}\right]$ \\
\hline $\mathbf{1 8}$ & 2.153 & 607 & -0.017 & 0.001 & 0.215 \\
$\mathbf{1 9}$ & 2.738 & 486 & -0.041 & 0.003 & 1.08 \\
$\mathbf{2 0}$ & 2.708 & 491 & -0.019 & 0.002 & 0.652 \\
$\mathbf{2 1}$ & 2.941 & 455 & -0.055 & 0.003 & 1.10 \\
$\mathbf{2 2}$ & 2.987 & 448 & -0.017 & 0.002 & 0.805 \\
\hline
\end{tabular}

Consequently, we decided to focus on $\mathbf{2 1}$ because, of all these four structures, it offers the best property trade-off and studied all distinct monosubstituted analogues with the same set of 18 substituents used with the azaphenalenes, as depicted in Scheme 7. The corresponding property maps at the EOM-CCSD/cc-pVDZ level of theory are shown in Figure 6 . The results show that tuning of the singlet-triplet gap, fluorescence rate and vertical excitation energy can be achieved to a significant extent even with a single substitution.<smiles></smiles>

\begin{tabular}{cccccc}
\multicolumn{7}{c}{$\mathbf{R}$} \\
\hline$-\mathrm{Me}$ & $-\mathrm{NH}_{2}$ & $-\mathrm{OH}$ & $-\mathrm{F}$ & $-\mathrm{SH}$ & $-\mathrm{Cl}$ \\
$-\mathrm{Br}$ & $-\mathrm{NHMe}$ & $-\mathrm{CHCH}_{2}$ & $-\mathrm{C}(\mathrm{O}) \mathrm{H}$ & $-\mathrm{CCH}$ & $-\mathrm{NC}$ \\
$-\mathrm{CN}$ & $-\mathrm{NMe}_{2}$ & $-\mathrm{C}(\mathrm{O}) \mathrm{Me}$ & $-\mathrm{S}(\mathrm{O}) \mathrm{Me}$ & $-\mathrm{NO}_{2}$ & $-\mathrm{CF}_{3}$
\end{tabular}

Scheme 7. Systematic monosubstitution of compounds $\mathbf{2 1}$ with diverse substituents. 
a)

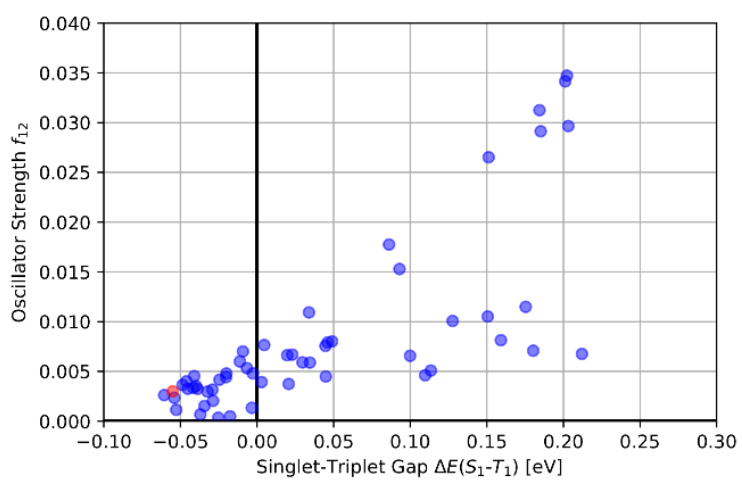

b)

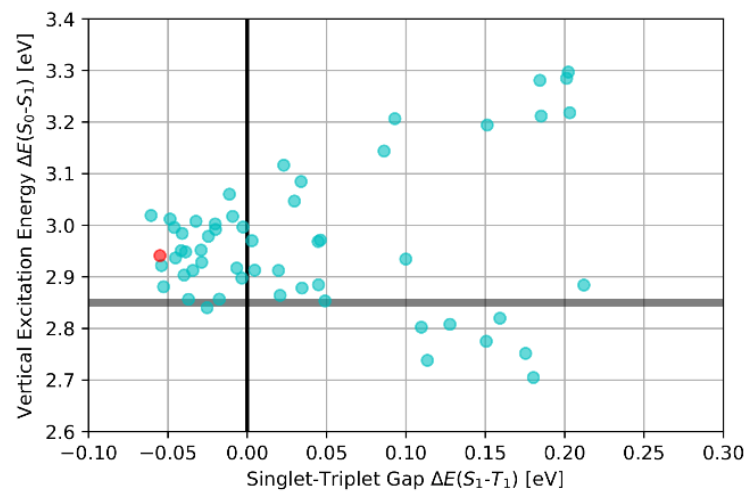

Figure 6. Maps of singlet-triplet gaps, oscillator strengths and vertical excitation energies of monosubstituted analogues of $\mathbf{2 1}$ with the substituents shown in Scheme 7 at the EOM-CCSD/cc-pVDZ level of theory. The red data point corresponds to unsubstituted 21 . The horizontal gray line in (b) indicates a vertical excitation energy of $2.85 \mathrm{eV}$ corresponding to about $468 \mathrm{~nm}$, after correcting for the solvatochromic shift.

Having identified $\mathbf{2 1}$ as the most promising azacyclopenta[cd]phenalene core structure and studied the effect of small substituents on its properties, we carried out stepwise systematic structural modifications of $\mathbf{2 1}$ to optimize for INVEST molecules with inverted singlet-triplet gaps, appreciable oscillator strengths and vertical excitation energies suitable for blue emitters. Hence, three target properties were optimized simultaneously. The optimization progress is illustrated in Figure 7. Again, important structures along the optimization trajectory are marked with diamond markers in Figure 7ab, with red markers in Figure 7c-d, and highlighted in Table 5 . These results show that blue INVEST emitters can very likely be realized, and they demonstrate again that INVEST molecules with appreciable fluorescence rates are likely more common than expected previously. 
a)

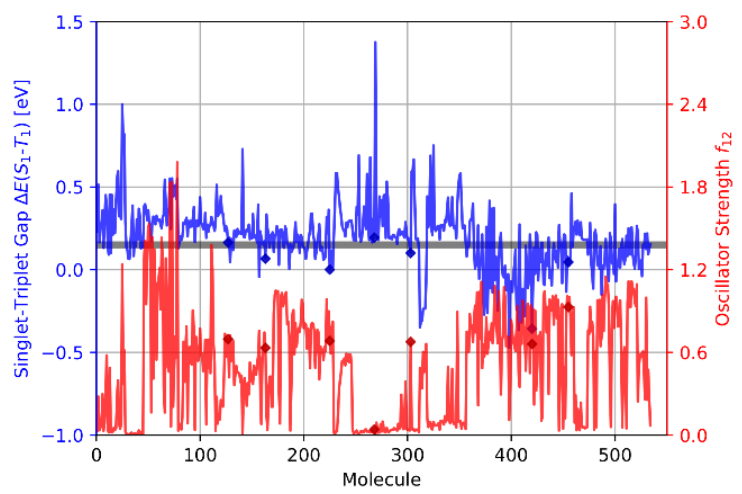

c)

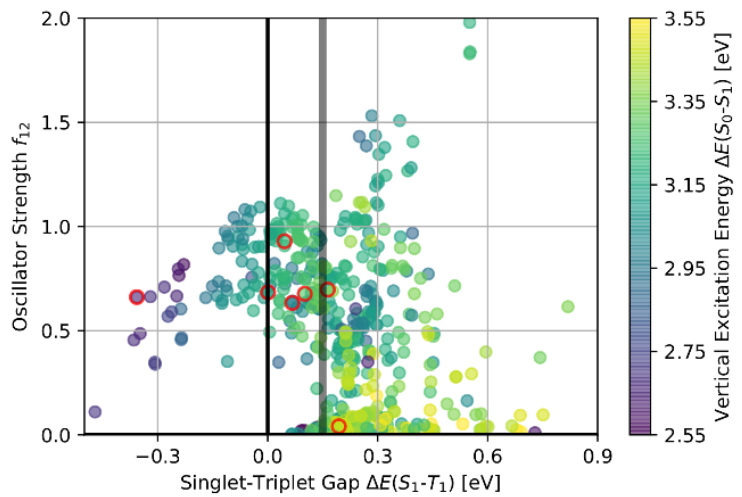

b)

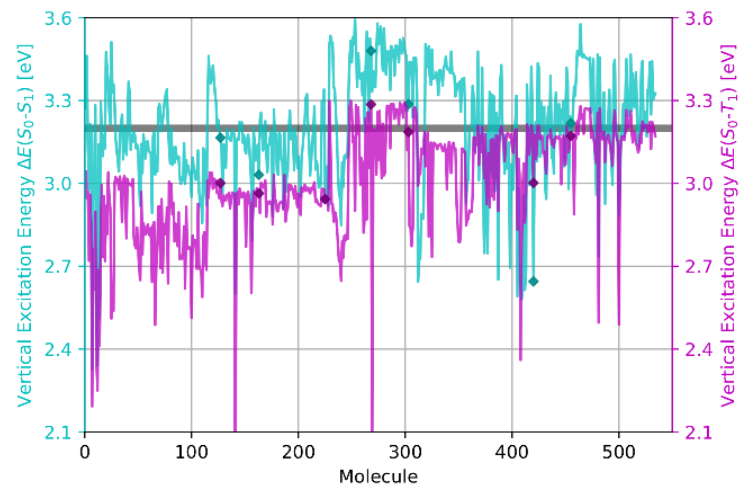

d)

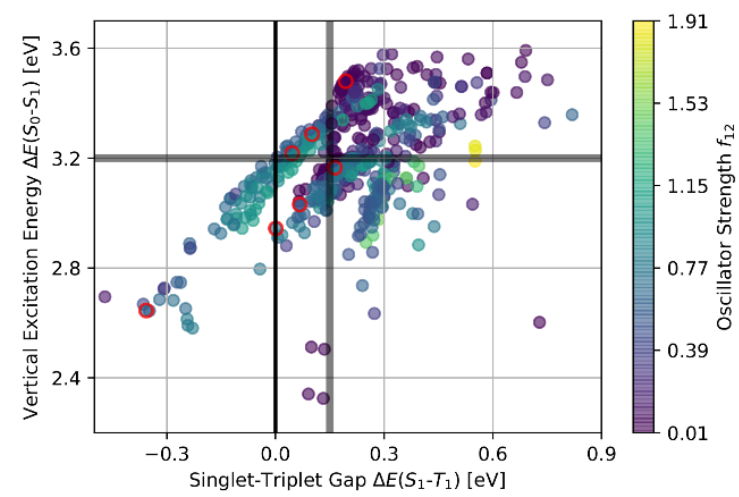

Figure 7. Optimization progress for singlet-triplet gap and oscillator strength (a), vertical $\mathrm{S}_{1}$ and $\mathrm{T}_{1}$ excitation energies (b), and property maps (c-d) of all compounds investigated during the optimization, aiming at potential blue INVEST emitters. Important structures are marked with diamond markers (ab) and red marker outlines (c-d), respectively. The horizontal gray line in (b) and (d) indicates a vertical excitation energy of $3.2 \mathrm{eV}$ corresponding to about $448 \mathrm{~nm}$, after correcting for the solvatochromic shift.

Table 5. Important structures along the optimization trajectory, aimed at potential blue INVEST emitters, and their properties. Absorption wavelengths, $\lambda\left(\mathrm{S}_{0}-\mathrm{S}_{1}\right)$, are corrected based on experimental data (vide supra, details in the Supporting Information).

\begin{tabular}{|c|c|c|c|c|c|}
\hline Compound & $\begin{array}{c}\Delta \mathrm{E}\left(\mathrm{S}_{0}-\mathrm{S}_{1}\right) \\
{[\mathrm{eV}]}\end{array}$ & $\begin{array}{c}\lambda\left(\mathrm{S}_{0}-\mathrm{S}_{1}\right) \\
{[\mathrm{nm}]}\end{array}$ & $\begin{array}{c}\Delta \mathrm{E}\left(\mathrm{S}_{1}-\mathrm{T}_{1}\right) \\
{[\mathrm{eV}]}\end{array}$ & $f_{12}$ & $\mathrm{k}_{\mathrm{F}}\left[10^{6} \mathrm{~s}^{-1}\right]$ \\
\hline & 3.165 & 453 & 0.164 & 0.697 & 303 \\
\hline 24 & 3.031 & 473 & 0.067 & 0.633 & 252 \\
\hline & 2.944 & 488 & 0.001 & 0.684 & 257 \\
\hline
\end{tabular}




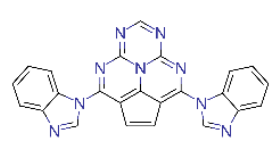

26

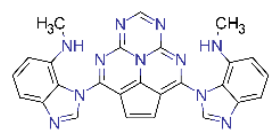

27

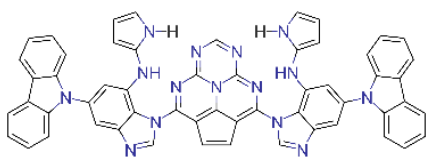

28

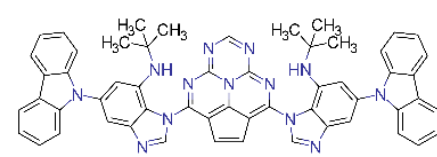

29
3.480

412

0.194

0.041

21.5

3.287

436

0.101

0.677

2.645

543

$-0.357$

0.661

201

3.218

446

0.046

0.929

417

Validation of Optimized Structures. To validate our findings, we decided to use minimal analogues of promising structures identified above and confirm their properties using higher-level of theory. Furthermore, we evaluated vibrational contributions to the singlet-triplet gaps as above and tested for the possibility of excited-state intramolecular proton transfer (ESIPT) ${ }^{102-110}$ in our hydrogen-bonded INVEST molecules. The minimal analogues selected are defined in Scheme 8. The results of high-level theory methods, as well as the comparison between Franck-Condon (vertical) and minima-to-minima (adiabatic) singlet-triplet gaps, are illustrated in Figure 8. The benchmark methods depicted in Figure $8 a$ confirm the significant increase in oscillator strength obtained while (largely) maintaining the inverted gaps, as observed at the $\omega B$ 2PLYP/def2-SVP level of theory. Notably, the minimal analogues we selected for validation are neither the best candidates we found in terms of inverted singlet-triplet gaps nor in terms of fluorescence rates, yet they still show promise for use as INVEST emitters in applications. Furthermore, Figure $8 \mathrm{~b}$ shows that vibrational contributions to the singlet-triplet gap are generally negligible for the minimal analogues selected. We observed the largest adverse vibrational effect for compound $\mathbf{4 1}$, but it still amounts only to $0.06 \mathrm{eV}$.

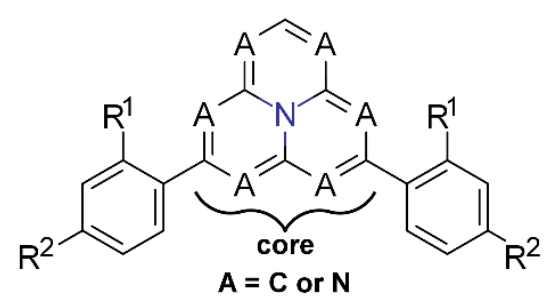

\begin{tabular}{cccccccc} 
Compound & Core & $\mathbf{R}^{\mathbf{1}}$ & $\mathbf{R}^{\mathbf{2}}$ & Compound & Core & $\mathbf{R}^{\mathbf{1}}$ & $\mathbf{R}^{\mathbf{2}}$ \\
\hline $\mathbf{3 0}$ & $\mathbf{3}$ & $\mathrm{H}$ & $\mathrm{H}$ & $\mathbf{3 8}$ & $\mathbf{5}$ & $\mathrm{H}$ & $\mathrm{H}$ \\
$\mathbf{3 1}$ & $\mathbf{3}$ & $\mathrm{NH}_{2}$ & $\mathrm{H}$ & $\mathbf{3 9}$ & $\mathbf{5}$ & $\mathrm{NH}_{2}$ & $\mathrm{H}$ \\
32 & $\mathbf{3}$ & $\mathrm{H}$ & $\mathrm{NH}_{2}$ & $\mathbf{4 0}$ & $\mathbf{5}$ & $\mathrm{H}$ & $\mathrm{NH}_{2}$ \\
33 & $\mathbf{3}$ & $\mathrm{NH}_{2}$ & $\mathrm{NH}_{2}$ & 41 & $\mathbf{5}$ & $\mathrm{NH}_{2}$ & $\mathrm{NH}_{2}$ \\
34 & 4 & $\mathrm{H}$ & $\mathrm{H}$ & $\mathbf{2 3}$ & $\mathbf{6}$ & $\mathrm{H}$ & $\mathrm{H}$ \\
35 & 4 & $\mathrm{NH}_{2}$ & $\mathrm{H}$ & 42 & 6 & $\mathrm{NH}_{2}$ & $\mathrm{H}$ \\
36 & 4 & $\mathrm{H}$ & $\mathrm{NH}_{2}$ & 43 & 6 & $\mathrm{H}$ & $\mathrm{NH}_{2}$ \\
37 & 4 & $\mathrm{NH}_{2}$ & $\mathrm{NH}_{2}$ & 44 & 6 & $\mathrm{NH}_{2}$ & $\mathrm{NH}_{2}$
\end{tabular}

Scheme 8. Minimal analogues of INVEST molecules with appreciable fluorescence rates used for validation. 
a)

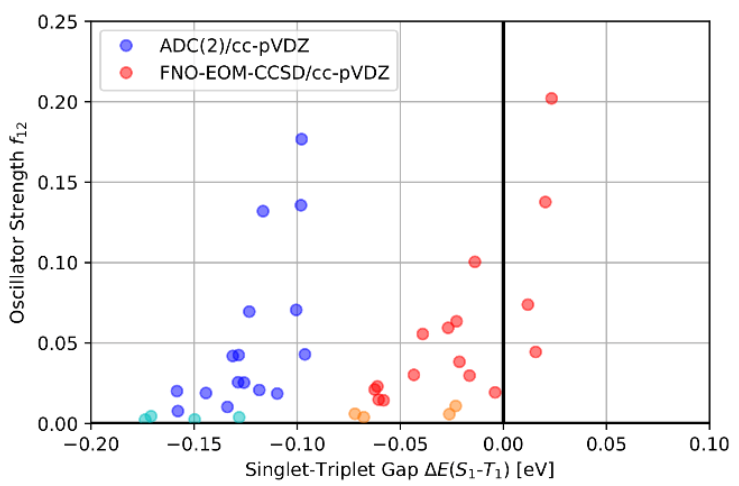

b)

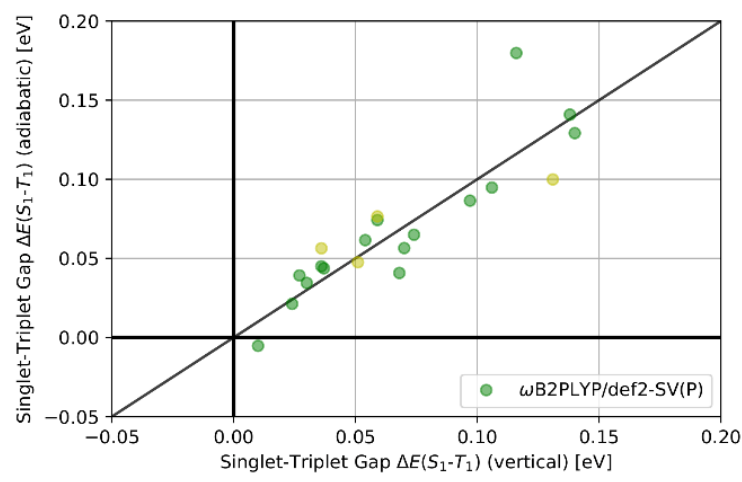

Figure 8. Validation of minimal analogues of INVEST molecules with appreciable fluorescence rates with benchmark quality methods (a) and comparison of their vertical and adiabatic singlet-triplet gaps (b). Data points with lighter colors correspond to the corresponding unsubstituted cores 3-6.

Finally, we tested the possibility of ESIPT in all validation compounds with intramolecular hydrogen bonds, namely $\mathbf{3 1}, \mathbf{3 3}, \mathbf{3 5}, \mathbf{3 7}, \mathbf{3 9}, \mathbf{4 1}, \mathbf{4 2}$ and $\mathbf{4 4}$. We tested for both single and double proton transfer from the aniline to the respective hydrogen-bonded core nitrogen atom by displacing the hydrogen atom accordingly and optimizing the resulting structures in the $\mathrm{S}_{0}, \mathrm{~S}_{1}$ and $\mathrm{T}_{1}$ manifolds, respectively. The corresponding results are provided in Table 6. For almost all compounds, neither single (1 PT), nor double (2 PT) proton transfer results in a stable state in the $S_{1}$ manifold as geometry optimization reversed the proton transfer(s) back to the original structures. In the $S_{0}$ manifold, proton transfer never resulted in a stable state. In the $\mathrm{T}_{1}$ manifold, single proton transfer generally resulted in stable states, which were energetically uphill for all validation compounds except $\mathbf{4 2}$. Nevertheless, for $\mathbf{4 2}$, single proton transfer was energetically downhill only by about $0.08 \mathrm{eV}$. Double proton transfer resulted in a stable state in the $T_{1}$ manifold only for 44 . Hence, ESIPT is unlikely to cause significant property changes to the INVEST molecules studied in this paper.

Table 6. Test for excited-state intramolecular proton transfer (ESIPT) in minimal analogues of INVEST molecules with appreciable fluorescence rates. The table entries provide the energy differences of the proton transfer states (PT) to the corresponding initial states in the respective state manifolds $\left(S_{0}, S_{1}\right.$ or $\mathrm{T}_{1}$ ) at the $\omega \mathrm{B} 2 \mathrm{PLYP} /$ def2-SV(P) level of theory. Unstable structures, denoted as "-," showed reverse proton transfer during geometry optimization.

\begin{tabular}{ccccccc}
\hline \multirow{2}{*}{ Compound } & \multicolumn{2}{c}{$\mathrm{E}\left(\mathbf{S}_{\mathbf{0}}\right)[\mathrm{eV}]$} & \multicolumn{2}{c}{$\mathrm{E}\left(\mathrm{S}_{1}\right)[\mathrm{eV}]$} & \multicolumn{2}{c}{$\mathrm{E}\left(\mathrm{T}_{1}\right)[\mathrm{eV}]$} \\
& $\mathbf{1} \mathbf{P T}$ & $\mathbf{2} \mathbf{P T}$ & $\mathbf{1} \mathbf{P T}$ & $\mathbf{2} \mathbf{P T}$ & $\mathbf{1 ~ P T}$ & $\mathbf{2} \mathbf{P T}$ \\
\hline $\mathbf{3 1}$ & - & - & - & - & +0.49 & - \\
$\mathbf{3 3}$ & - & - & - & - & +0.62 & - \\
$\mathbf{3 5}$ & - & - & - & - & +0.34 & - \\
$\mathbf{3 7}$ & - & - & - & - & +0.56 & - \\
$\mathbf{3 9}$ & - & - & +0.86 & - & +0.05 & - \\
$\mathbf{4 1}$ & - & - & - & - & +0.31 & - \\
$\mathbf{4 2}$ & - & - & - & - & -0.08 & - \\
$\mathbf{4 4}$ & - & - & - & - & +0.11 & +0.98 \\
\hline
\end{tabular}

\section{Discussion}

Overall, we find that modification of phenalene cores results in a rich space of INVEST molecules as the singlet-triplet gap, the fluorescence rate and the absorption wavelength can be tuned over wide property intervals. We also discover that INVEST molecules with appreciable fluorescence rate are not 
only possible, but readily derived by careful substitution of azaphenalenes. Finally, we establish that INVEST molecules with appreciable fluorescence rate with azaphenalene cores cover the entire visible light spectrum showing promise as organic electronic materials in general, especially for emissive materials. In the following discussion, we will investigate these claims in detail, and discuss the potential of INVEST molecules as OLED materials by comparing their design and properties to established organic emitters.

Design Principles. The general principle of INVEST emitters compared to the current generations of OLEDs is illustrated in Scheme 9. First generation emitters are unable to harvest triplet excitons leading to a maximum internal quantum efficiency (IQE) of only $25 \%$, limiting external quantum efficiency (EQE) to about $5 \% .^{25,92,93,95,111}$ Phosphorescent emitters improve on that by enabling fast ISC allowing all singlet excitons to be down-converted to triplets and having comparably fast phosphorescence rate constants. . $5,92,93,95,111$ However, their main drawbacks are low long-term device stabilities due to high excited triplet populations causing triplet-triplet annihilation, singlet-triplet annihilation and tripletpolaron annihilation (which are the major cause of decomposition ${ }^{89,92,96}$ ), and high cost due to reliance on noble metals. ${ }^{25,95}$ The third generation of emissive materials, TADF emitters, solve these problems at least in part as they do not require noble metals and tend to have higher long-term device stabilities, while allowing efficient triplet exciton harvesting. ${ }^{25,89,97,112}$ Yet, stability is not ideal as excited triplet populations are still significant. ${ }^{89}$ Hyperfluorescence emitters are currently emerging as fourth generation of OLED materials. ${ }^{113}$ Their central idea is to combine the advantages of first-generation fluorescent emitters and third-generation TADF emitters by using a TADF assistant dopant to harvest both singlet and triplet excitons efficiently and utilizing Förster resonance energy transfer (FRET) to transfer all singlet excitons from the TADF assistant dopant to the fluorescent emitter. ${ }^{25,91,113}$ Subsequently, light is emitted from the first excited singlet of the fluorescent emitter. OLEDs based on hyperfluorescence, like TADF emitters, reach $100 \%$ internal quantum efficiencies, but have narrower emission leading to higher color purity and also have higher long-term device stability. ${ }^{113}$ However, it is important to realize that hyperfluorescence emitters are also compatible with INVEST emitters as assistant dopants potentially improving their performance even further. Hence, INVEST emitters have the potential to surpass all previous generations of emissive materials in terms of long-term device stability, especially for blue emitters, because the change in the relative energies of excited singlet and triplet leads to considerably lower triplet populations. Furthermore, they do not rely on noble metals either and harvest triplet excitons at least equally well. Some of the most important properties of typical emitters from these OLED generations are summarized in Table 7. 


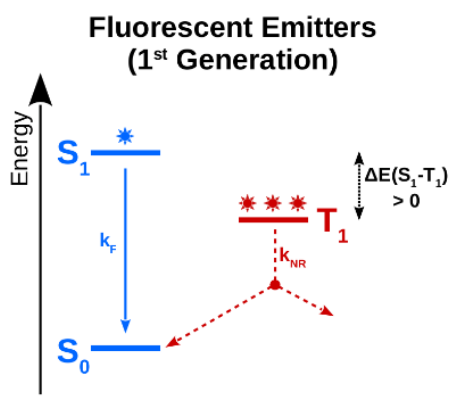

Phosphorescent Emitters ( $2^{\text {nd }}$ Generation)

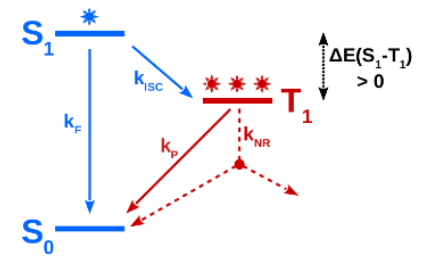

Hyperfluorescence Emitters

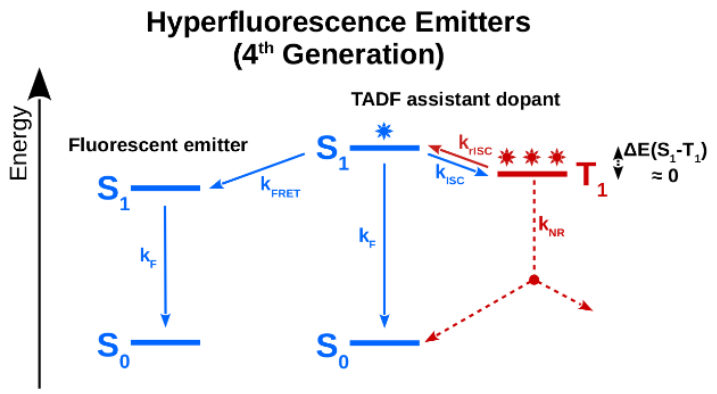

TADF Emitters ( $3^{\text {rd }}$ Generation)

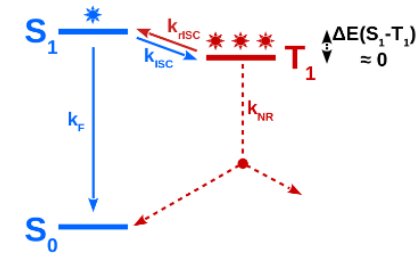

INVEST Emitters ( $5^{\text {th }}$ Generation)

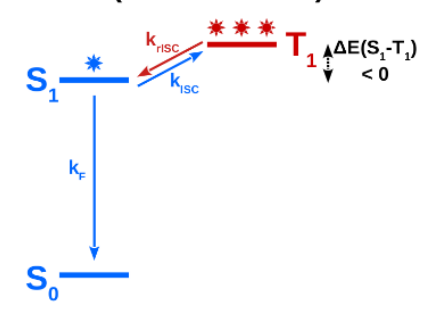

Scheme 9. Jablonski diagrams of previous and current generations of OLED materials compared to INVEST emitters as proposed next generation summarizing their key properties.

Table 7. Comparison of important properties of typical emitters from current generations of emissive materials with INVEST molecules.

\begin{tabular}{cccccc}
\hline Property & Fluorescent & Phosphorescent & TADF & Hyperfluorescent & INVEST \\
\hline $\begin{array}{c}\text { Internal quantum } \\
\text { efficiency (IQE) }\end{array}$ & $\leq 25 \%$ & $\leq 100 \%$ & $\leq 100 \%$ & $\leq 100 \%$ & $\leq 100 \%$ \\
$\begin{array}{c}\text { Composition } \\
\begin{array}{c}\text { Long-term } \\
\text { stability }\end{array}\end{array}$ & Organic & Organometallic & $\begin{array}{c}\text { Organic, } \\
\text { Organometallic }\end{array}$ & $\begin{array}{c}\text { Organic, } \\
\text { Organometallic }\end{array}$ & $\begin{array}{c}\text { Organic, } \\
\text { Organometallic? }\end{array}$ \\
\hline Cost & Low & Low & Medium & High & High? \\
\hline
\end{tabular}

Generally, the design of organic emitters has focused largely on minimizing singlet-triplet gaps based on a two-orbital two-electron model (TOTEM) using highest occupied molecular orbital (HOMO) and lowest unoccupied molecular orbital (LUMO). ${ }^{114-116}$ Within TOTEM, the singlet-triplet gap equals twice the exchange integral between HOMO and LUMO. ${ }^{114-116}$ Hence, based on that model, the singlet-triplet gap can be minimized by reducing the spatial overlap between HOMO and LUMO, and this strategy proved effective leading to various designs for TADF emitters. . $^{95,96,117}$ However, it has been shown recently that TOTEM is not sufficient to predict singlet-triplet gaps accurately, as the inclusion of additional states is necessary to account for the non-negligible contribution of double excitations. ${ }^{22,116}$ An alternative explanation for the missing physics is the absence of spin polarization in TOTEM. ${ }^{23,118-}$ ${ }^{121}$ It is important to point out that both double excitation contributions and spin polarization are equivalent mechanisms enabling inverted singlet-triplet gaps. The difference between the two concepts is that double excitation contributions are interpreted in the state description of electronic excitation, spin polarization is interpreted in the orbital description. ${ }^{23}$ Importantly, the neglect of double excitations makes inverted singlet-triplet gaps impossible within TOTEM. ${ }^{22,116}$ Therefore, refined models accounting for double excitations are required to describe the low-energy excited electronic states of INVEST emitters appropriately. TD-DFAs need to be double-hybrid or higher in their rung ${ }^{41,122}$ or have to be used as spin-flip variant with a well-behaved triplet state as reference. ${ }^{123}$ Accordingly, a four-state model has been proposed as minimal model to describe the low-energy 
excited states of INVEST molecules. ${ }^{22,116}$ It shows that, to design of INVEST emitters, the minimization of the spatial overlap between HOMO and LUMO is important but not sufficient and the increase of double excitation character are equally important. Importantly, increasing double excitation character is an effective mechanism to realize large fluorescence rates and small singlet-triplet gaps simultaneously. ${ }^{118,124}$

One main achievement in this work is breaking the empirical observation that molecules with very small singlet-triplet gaps tend to have very small oscillator strength, ${ }^{95}$ and effective illustrations of this observation have been provided in the context of high-throughput virtual screenings. ${ }^{116,125,126}$ INVEST molecules with appreciable oscillator strength were, to the best of our knowledge, previously unheard of. ${ }^{22}$ The trade-off between singlet-triplet gap and oscillator strength is a consequence of the small spatial overlap between HOMO and LUMO minimizing the corresponding exchange integral, but higher spatial overlap between HOMO and LUMO increases the oscillator strength of the respective electronic excitation. ${ }^{96,117,127}$ Nevertheless, the main difference is that spatially extended HOMOs and LUMOs lead to smaller singlet-triplet gaps, but to larger oscillator strengths. ${ }^{127,128}$ Thus, extending $\pi$-systems has been used successfully as a strategy to escape the empirically observed correlation between these properties, for instance, by introducing phenylene linkers between donor and acceptor moieties. ${ }^{96,127,129}$

As pointed out previously, 1 follows the general design strategy of TADF emitters using the multiple resonance effect (MRE), ${ }^{22}$ and this applies equally to all the INVEST molecules discovered in this study. MRE emitters rely on spatial HOMO-LUMO separation within a single $\pi$-system by introducing heteroatoms at appropriate positions to induce opposing resonance effects on neighboring atoms in the system to minimize exchange integrals and singlet-triplet gaps. ${ }^{130}$ These emitters were introduced as new design for TADF emitters only recently, ${ }^{130}$ and they have been shown to possess several advantages over the classic donor-acceptor design. In particular, such molecules have significantly higher fluorescence rates, small reorganization energies, and high color purity, due to their rigidity. ${ }^{130-}$ 132 Up to now, MRE emitters have not been reported on extensively despite showing promise for blue ${ }^{130,133-136}$ and green ${ }^{132,135,137}$ emitters, and as organic TADF laser dyes. ${ }^{138}$ One of their drawbacks compared to classical donor-acceptor (D-A) emitters are their typically larger singlet-triplet gaps. ${ }^{131,136,137}$ Nevertheless, MRE emitters were shown experimentally to harvest triplet excitons efficiently with IQEs of up to $100 \% \cdot{ }^{130}$ Importantly, all INVEST emitters with appreciable fluorescence rate discovered follow a donor-acceptor-donor (D-A-D) architecture with the acceptor being the INVEST core structure, with the main difference that twisted D-A connections are avoided. In the best performing candidates, the acceptor cores and the donors are separated by a $\pi$-system. For TADF emitters, this is one of the most common molecular architectures. ${ }^{112,117,139,140}$ Notably, this analysis reveals that all chemical moieties with inherent INVEST properties utilized so far are acceptors. Accordingly, this poses the question whether future studies could also find donor structures with inverted singlet-triplet gaps as this would increase the chemical space of potential INVEST emitters drastically and allow entirely different architectures. Furthermore, it also needs to be investigated whether INVEST emitters could be good candidates for electrically pumped organic lasers. ${ }^{141,142}$

The INVEST emitters discovered in this study solve one of the disadvantages of most previous MRE emitters by having inverted singlet-triplet gaps, which will make triplet exciton harvesting even more efficient and improve long-term device stability. Moreover, our INVEST emitter design benefits from all the advantages of MRE emitters. The high rigidity of the INVEST core structures is expected to produce narrow emission of high color purity, improved stability and increased EQE due to improved 
light outcoupling efficiency. ${ }^{96}$ Thus, these emitters can resolve the main drawbacks of classical TADF emitters based on the D-A design. ${ }^{96}$ In addition, we have found INVEST emitters with even higher oscillator strengths than the best performing small molecule MRE emitters reported so far, ${ }^{130,131,137}$ and specifically much brighter potential blue emitters, showing that high fluorescence rate constants can be realized with this design. Notably, the INVEST design of having largely co-planar molecules with high oscillator strength but small singlet-triplet gaps aligns perfectly with the criteria established in a recent account for promising new OLED emitter designs. ${ }^{143}$ Classical TADF emitters based on the D-A design typically have oscillator strengths for the first singlet excitation of typically only up to $0.1,116,143$ small molecule MRE emitters with values larger than 0.4 have been reported. ${ }^{130,131,137}$ In this study, we discovered INVEST emitters with predicted oscillator strengths larger than 1 . Hence, we believe that INVEST emitters based on the MRE design have the potential to surpass the performance of current TADF and phosphorescent emitters used in OLED devices and establish a new generation of organic electronic materials.

The inverted energy ordering of excited singlet and triplet will result in fundamentally different dynamic behavior between INVEST emitters and TADF emitters. Assuming fast thermal equilibration of the populations of first excited singlet and triplet states, the delayed fluorescence intensity would have an inverse temperature-dependence, i.e. higher temperatures lead to reduced delayed fluorescence due to an increasing triplet population. ${ }^{112}$ However, this inverse temperature dependence is extremely hard to detect because the changes in the rISC rates, i.e. transitions from first excited triplets to singlets, will be very small. In other words, in contrast to emissive TADF materials, INVEST emitters will have almost temperature-independent rISC rates but temperaturedependent ISC rates. ${ }^{144}$ Hence, the temperature-dependence of the delayed fluorescence intensity is not a robust experimental diagnostic for INVEST emitters. Accordingly, delayed fluorescence from INVEST emitters is not thermally activated as triplet excitons are downconverted to singlet excitons and do not require (significant) thermal energy. Hence, defining INVEST as new class of emitters, distinct from TADF emitters, is justified.

Importantly, derivatives of $\mathbf{2}$ have been tested as potential OLED emitters in devices already several years ago. ${ }^{145-147}$ However, it has not been recognized until very recently that these derivatives likely have an inverted singlet-triplet gap, which is in full agreement with a recent combined computational and experimental study on this molecule class. ${ }^{23}$ It was even reported that one of these derivatives shows the opposite temperature-dependence of the delayed fluorescence rate compared to TADF molecules but it was not interpreted as possibly resulting from an inverted singlet-triplet gap. ${ }^{145} \mathrm{We}$ used our computational protocol on these structures and the results indeed suggest inverted singlettriplet gaps (Details in the SI). Altogether, these early reports are evidence that INVEST molecules can indeed be efficient emissive materials for OLED devices. Importantly, one of the reasons that derivatives of $\mathbf{2}$ were not pursued further as organic emitters was their comparably modest performance caused by low internal quantum efficiencies. This is likely caused by their negligible fluorescence rates as the corresponding electronic transitions are symmetry-forbidden. ${ }^{23}$ Hence, we believe that INVEST molecules with appreciable fluorescence rates will improve significantly on their performance as emissive materials and will unlock their true potential. Furthermore, compound $\mathbf{3 8}$ and several other derivatives of $\mathbf{5}$ have been synthesized very recently and their optical properties characterized. ${ }^{83-86}$ Based on our computational results, $\mathbf{3 8}$ has an inverted singlet-triplet gap and appreciable fluorescence rate. While the latter has been confirmed experimentally, ${ }^{86}$ the inverted singlet-triplet gap has remained unrecognized, as the use of double-hybrid TD-DFAs capable to characterize their electronic properties is not widespread. Experimental attempts to estimate its 
singlet-triplet gap were not reported ${ }^{86}$ These studies demonstrate that at least some of the molecules investigated in this study are readily synthesizable making them promising targets for experimental follow-up studies.

Another crucial design strategy we discovered is the use of strong intramolecular hydrogen bonds between primary or secondary amines and nitrogen lone pairs of the INVEST cores to rigidify the molecules, control their conformations and increase their fluorescence rates significantly without a concomitant increase in singlet-triplet gap. The increased rigidity will likely contribute to narrow emission bands leading to high color purity, which is important for display applications. ${ }^{130,143}$ It should be noted that a similar strategy to increase the fluorescence rate using boron complexation was demonstrated to be effective on substituted derivatives of 5 very recently. ${ }^{85}$ However, the use of these strong intramolecular hydrogen bonds comes with the risk of potential ESIPT, which could introduce low-lying triplet states and break the INVEST property. ${ }^{102-110}$ Nevertheless, in the set of validation compounds only $\mathbf{4 2}$ shows proton transfer to lead to a lower excited triplet state causing an increase of the singlet-triplet gap of $0.08 \mathrm{eV}$. Both the observations that only one of the investigated structures shows this detrimental effect and that the increase in $\mathbf{4 2}$ merely is $0.08 \mathrm{eV}$ demonstrate that, at least molecules with comparable structures, ESIPT is unlikely to cause a significant deterioration of the molecular properties of INVEST emitters. Thus, we believe that strong intramolecular hydrogen bonds are a promising molecular design strategy for organic emissive materials.

Finally, one open question about INVEST emitters based on the MRE design is whether rISC can be efficient in these systems. Even though it is energetically favorable to undergo rISC from excited triplets to singlets, this process is not necessarily fast. Recently, the excited state dynamics of typical MRE emitters have been investigated independently in two computational studies to gain insight into the ISC and rISC mechanisms. ${ }^{148,149}$ It was found that the general mechanisms for the studied MRE emitters are equivalent to other organic TADF emitters and proceed via second-order spin-vibronic coupling. ${ }^{148,150-154}$ However, because of the inverted state ordering, the higher excited triplet states will likely not mediate ISC and rISC for INVEST emitters because the associated energy differences are even larger. Hence, we expect that direct spin-orbit coupling between $S_{1}$ and $T_{1}$ is the most likely mechanism for these processes. For TADF emitters based on the MRE design, it was proposed that a decrease in the singlet-triplet gap will lead to an acceleration of rISC. ${ }^{148}$ Hence, INVEST emitters based on the same design with singlet-triplet gaps small in magnitude will likely have a higher rISC rate compared to TADF emitters based on the MRE design, and these processes will likely proceed via direct coupling between $S_{1}$ and $T_{1}$ as these are close in energy. INVEST emitters with singlet-triplet gaps large in magnitude will likely have slower rISC rates due to the energetic distance between the interconverting states, but higher energy singlet states close in energy could facilitate these processes. ${ }^{15}$ Alternatively, substituents on the INVEST emitters could enable new ISC and rISC pathways via selected vibrational modes but this needs to be probed on specific structural classes of molecules. Overall, this suggests rISC likely to be efficient in the INVEST emitters discovered as many of them have singlet-triplet gaps small in magnitude. However, follow-up studies investigating their excited state dynamics need to be carried out to gain more insight into the underlying mechanisms, and future INVEST emitters need to be designed with maximizing rISC rate constants as explicit target.

Benchmarking and Validation. Currently, classification of INVEST molecules relies largely on using various reliable $a b$ initio computational methods ${ }^{22,23}$ because experimental evidence is sparse and hard to obtain. ${ }^{15,23}$ To the best of our knowledge, there are only two published direct attempts of estimating an inverted singlet-triplet gap experimentally based on energy-transfer rates using known triplet 
sensitizers as references and they were carried out for $\mathbf{1}$ and another azaphenalene derivative. ${ }^{15,16}$ Yet, in both cases they are not fully conclusive as the associated uncertainties cannot exclude small but positive values with sufficient confidence. ${ }^{15,16}$ Furthermore, a recent study attempted to obtain spectroscopic evidence for the predicted inverted singlet-triplet gap in $2 .{ }^{23}$ Despite in-depth optical characterization of $\mathbf{2}$ including time-dependent photoluminescence decay measurements, with and without the presence of molecular oxygen as triplet quencher or external heavy atoms to facilitate ISC, and microsecond transient absorption measurements, it could only be concluded that no evidence for the involvement of a low energy triplet in the excited state dynamics probed has been found. ${ }^{23}$ This shortcoming needs to be addressed in future studies and reliable experimental methods for the determination of singlet-triplet gaps suitable for INVEST emitters need to be developed. Accordingly, the most reliable evidence for inverted singlet-triplet gaps still comes from ab initio characterization. Many well-established computational methods, all based on different theoretical models and assumptions, predict negative singlet-triplet gaps for both 1 and $2 .^{22,23}$ These methods all have in common that they account for contributions of double excitations. Hence, they also support the minimal four-state model developed recently describing singlet-triplet gaps of organic emitters with the correct physics. ${ }^{116}$

Based on comprehensive benchmarking against $A D C(2),{ }^{44-50} \mathrm{EOM}^{-C_{C S D}}{ }^{51-55}$ and FNO-EOM-CCSD ${ }^{51-59}$ calculations as references, we established a robust computational protocol using $\omega B 2 \mathrm{PLP},{ }^{41}$ a doublehybrid TD-DFA, ${ }^{68-71,122}$ as our computational workhorse (Figure 3). It accounts for double excitations perturbatively based on a $\mathrm{CIS}(\mathrm{D})^{155,156}$ calculation, and it was shown to be one of the most reliable TDDFA methods currently available. ${ }^{122}$ Generally, it reproduces trends in both singlet-triplet gaps and oscillator strengths faithfully. Occasional outliers of singlet-triplet gaps lead to missing molecules that would be inverted, but not to misidentifying ordinary structures as INVEST molecules (Figure 3a). The observed outliers for oscillator strength would only lead to under- rather than overestimation demonstrating that all INVEST molecules with appreciable oscillator strength identified based on $\omega B 2 P L P$ are very likely robust predictions (Figure $3 b$ ). Moreover, these outliers in predicted oscillator strength likely stem from EOM-CCSD as both ADC(2) and $\omega B 2$ PLP show excellent agreement with each other without notable outliers. The Gaussian process regression we performed (Figure 3a) could be applied for correcting predicted singlet-triplet gaps to get more accurate values. However, as we were mostly interested in testing for negative singlet-triplet gaps, correcting for the systematic offset between a zero gap at the EOM-CCSD level and the corresponding gap at the $\omega \mathrm{B} 2 \mathrm{PLP}$ level is sufficient.

There are several other approximations in our computational protocol that need to be addressed. First, most singlet-triplet gaps and fluorescence rate estimates are based on the Franck-Condon point of the molecules, which is likely reasonable as most of the structures are rigid. Accordingly, excited state geometry optimizations did not reveal any significant deterioration of the negative singlet-triplet gaps in the validation set. Additionally, color estimates were also based on the Franck-Condon point and disregard the Stokes shift, which is a very crude approximation. Moreover, color estimates were based on a calibration of computation against experimental spectra in solution. Due to the sparsity of suitable experimental data, the spectra used were not recorded in the same solvent (Details in the SI). Nevertheless, since the first excited singlets in the color benchmark set have largely locally excited character, the solvatochromic shift is not expected to be strongly solvent-dependent, ${ }^{23,130}$ and, at least in the experimental data set available, the data from multiple solvents seem to be consistent. Hence, the solvatochromic shift estimate used is an acceptable approximation, and serves to correct for a systematic offset in the vertical excitation energies in the gas phase obtained using $\omega B 2 P L P$. Yet, most experimental data points were in the middle of the visible light spectrum making the corrected values 
both in the low and high energy regions less robust, which is particularly relevant for potential blue emitters as their predicted vertical excitation energies estimates have the highest uncertainty. For future computational protocols, the impact of excited state geometry relaxation needs to be computed explicitly to find better candidates for INVEST emitters and obtain more reliable color estimates. Furthermore, experimental verification of predicted absorption and emission energies are also necessary.

Finally, the validation of trends in singlet-triplet gaps and oscillator strengths also requires further attention. While the respective results confirm the trends observed in the optimizations, the expensive computational methods necessary limit us to rather limited molecular sizes. Hence, future computational studies on larger and more realistic INVEST emitters need to be carried out to validate our findings. To do that, alternative computational approaches need to be tested, especially local excited state methods like LADC(2) or LCC2, to compute even larger molecules and also perform excited state geometry optimizations very reliably and efficiently. ${ }^{157-163}$ Additionally, systematic exploration of potential conical intersections between $\mathrm{S}_{0}, \mathrm{~S}_{1}$ and $\mathrm{T}_{1}$ states has only been carried out for $2^{23}$ and needs to be performed on more promising INVEST emitters as well to exclude any detrimental effects on IQE. Nevertheless, all the validations support our predictions and show that these molecules are likely good candidates for efficient organic emissive materials.

Effect of Core Structure and Substitution. The observation that systematic substitution of C-H by N in 1 and $\mathbf{1 8}$ leads to many new INVEST molecules partly supports the prediction made in a very recent paper that the inverted singlet-triplet gap is an intrinsic property of isoelectronic compounds of $2 .{ }^{164}$ However, the statement proves to be too optimistic as a significant fraction of these derivatives lose the inverted singlet-triplet gap as demonstrated in Figure 1 and Figure 5. We also realized that the introduction of an increasing number of nitrogen atoms at appropriate positions leads to a gradual increase of the vertical excitation energy of the first excited singlet. This has already been demonstrated in the 1980s based on a compilation of experimental data on a small subset of all the possible derivatives. ${ }^{15}$ This intrinsic feature of the azaphenalene core makes these derivatives very attractive for applications as emissive materials because a wide color spectrum is accessible without changing the molecular structures drastically. It enabled us to find potential INVEST emitters having various excitation energies with minimal effort, and it will facilitate further design. The observation that minimal modification of azaphenalenes leads to azacyclopenta[cd]phenalenes with significantly increased vertical excitation energies expands the accessible colors leading to reasonable coverage of the visible light spectrum by making efficient blue emitters attainable. It demonstrates that minimal modifications of the azaphenalene core can lead to large property changes and, hence, encourages the exploration of further derivatives in future studies. Importantly, the introduction of one additional 5-membered ring does not change the electronic properties completely as the inverted singlet-triplet gap is retained, and the impact of nitrogen substitution is comparable. However, while systematic substitution of $\mathrm{C}-\mathrm{H}$ by $\mathrm{N}$ is trivial in computational studies, it can strongly impact synthesizability. While substituted analogs of $\mathbf{5}$ have good literature precedence, ${ }^{72,75,76,81-86}$ derivatives of $\mathbf{3}, \mathbf{4}$ and $\mathbf{6}$ are less common. ${ }^{72-74,77,78,80,165}$ Moreover, to the best of our knowledge, neither $\mathbf{2 1}$ itself nor any substituted analogs have been synthesized before, only derivatives of $\mathbf{1 8}$ have been reported. ${ }^{99-101}$ Yet absence of literature precedence does not imply synthetic inaccessibility and we believe that our study will spark renewed interest in these compound classes, and motivate research groups to develop efficient synthesis routes for them. 
One recurring observation from the systematic exploration of derivatives of 1 and $\mathbf{1 8}$, including both the nitrogen substitution and the introduction of functional groups, is a connection between singlettriplet gap and oscillator strength. There is no strong correlation between these properties, but there is a trade-off that needs to be overcome as shown in the property maps. Selecting cores with the best trade-off between singlet-triplet gap and oscillator strength leads to an increase in estimated fluorescence rate constants of up to 3 orders of magnitude relative to 1 showing that the choice of core structure is crucial. This is also supported by the observation that the impact of substituents can be very different depending on the structure. For instance, while 1 shows only very modest changes in oscillator strength upon substitution, substituted analogs of 2-6 lead to significant changes. Moreover, we also observed a correlation between the vertical excitation energies and singlet-triplet gaps in substituted analogs of $\mathbf{2 1}$ as substituents affect excited singlet and triplet states differently. This was crucial during the optimization of blue INVEST emitters to find structural modifications that increase both excited singlet and triplet energies equivalently in order not to negatively impact the singlettriplet gaps.

\section{Conclusions}

In this work, we have explored organic molecules with inverted singlet-triplet gaps based on nitrogensubstituted phenalenes computationally. Through careful substitution of azaphenalenes with a combination of $\pi$-substituents, donor, and acceptor groups, we revealed a vast space of INVEST molecules with appreciable fluorescence rates. Additional modification of the phenalene core lead to the discovery of azacyclopenta[cd] phenalenes as promising blue INVEST emitters with considerable fluorescence rate. Most of these molecules are likely synthetically accessible and offer various advantages for optoelectronic applications, including potentially fast reverse intersystem crossing, increased device lifetime and high color purity.

Additionally, the full potential of INVEST molecules has yet to be unleashed because the chemical space exploration was far from exhaustive. For a more comprehensive exploration, systematic computerguided multi-objective optimization procedures are required, and we are already implementing this approach for future studies. Nevertheless, the large number of discovered structures suffices as a proof of concept for the existence of molecules with inverted singlet-triplet gaps and appreciable fluorescence rates. Importantly, the computational predictions need to be verified experimentally and work towards synthesizing and testing the most promising INVEST emitters is already underway in our lab. We envision that organic INVEST emitters have the potential to become the next generation of OLED materials.

\section{Associated Content}

Supporting Information

Details of data evaluation and calibration (PDF).

Structures of all explored compounds and corresponding computational results (PDF).

Cartesian coordinates of all optimized structures (XYZ). 


\section{Author Information}

Corresponding Author

alan@aspuru.edu

\section{Acknowledgments}

P. F. has received funding from the European Union's Horizon 2020 research and innovation program under the Marie Sklodowska-Curie grant agreement no. 795206. We acknowledge the Defense Advanced Research Projects Agency (DARPA) under the Accelerated Molecular Discovery Program under Cooperative Agreement No. HR00111920027 dated August 1, 2019. The content of the information presented in this work does not necessarily reflect the position or the policy of the Government. A. A.-G. thanks Dr. Anders G. Frøseth for his generous support. A. A.-G. also acknowledges the generous support of Natural Resources Canada and the Canada 150 Research Chairs program. We thank Compute Canada for providing computational resources.

\section{References}

(1) Hund, F. Z. Physik 1925, 33, 345-371.

(2) Koseki, S.; Nakajima, T.; Toyota, A. Can. J. Chem. 1985, 63, 1572-1579.

(3) Kutzelnigg, W.; Morgan, J. D. Z Phys D - Atoms, Molecules and Clusters 1996, 36, 197214.

(4) Jablonski, A. Nature 1933, 131, 839-840.

(5) Valeur, B.; Berberan-Santos, M. N. J. Chem. Educ. 2011, 88, 731-738.

(6) Farr, E. P.; Quintana, J. C.; Reynoso, V.; Ruberry, J. D.; Shin, W. R.; Swartz, K. R. J. Chem. Educ. 2018, 95, 864-871.

(7) Leermakers, P. A.; Vesley, G. F. J. Chem. Educ. 1964, 41, 535.

(8) Swenton, J. S. J. Chem. Educ. 1969, 46, 7.

(9) Miller, J. B. J. Chem. Educ. 1999, 76, 592.

(10) Demas, J. N. J. Chem. Educ. 1983, 60, 803.

(11) Richards, J. H. J. Chem. Educ. 1968, 45, 398.

(12) Jaffe, H. H.; Miller, A. L. J. Chem. Educ. 1966, 43, 469.

(13) Toyota, A.; Nakajima, T. Journal of the Chemical Society, Perkin Transactions 2 1986, $0,1731-1734$.

(14) Toyota, A. Theoret. Chim. Acta 1988, 74, 209-217.

(15) Leupin, W.; Wirz, J. J. Am. Chem. Soc. 1980, 102, 6068-6075.

(16) Leupin, Werner.; Magde, Douglas.; Persy, Gabriele.; Wirz, Jakob. J. Am. Chem. Soc. 1986, 108, 17-22.

(17) Olivier, Y.; Sancho-Garcia, J.-C.; Muccioli, L.; D’Avino, G.; Beljonne, D. J. Phys. Chem. Lett. 2018, 9, 6149-6163.

(18) Difley, S.; Beljonne, D.; Van Voorhis, T. J. Am. Chem. Soc. 2008, 130, 3420-3427.

(19) Eizner, E.; Martínez-Martínez, L. A.; Yuen-Zhou, J.; Kéna-Cohen, S. Science Advances 2019, 5, eaax4482.

(20) Olivier, Y.; Yurash, B.; Muccioli, L.; D’Avino, G.; Mikhnenko, O.; Sancho-García, J. C.; Adachi, C.; Nguyen, T.-Q.; Beljonne, D. Phys. Rev. Materials 2017, 1, 075602.

(21) Trujillo, C.; Sánchez-Sanz, G.; Alkorta, I.; Elguero, J. Struct Chem 2017, 28, 345-355.

(22) de Silva, P. J. Phys. Chem. Lett. 2019, 10, 5674-5679.

(23) Ehrmaier, J.; Rabe, E. J.; Pristash, S. R.; Corp, K. L.; Schlenker, C. W.; Sobolewski, A. L.; Domcke, W. J. Phys. Chem. A 2019, 123, 8099-8108.

(24) Reid, D. H. Q. Rev. Chem. Soc. 1965, 19, 274-302. 
(25) Wong, M. Y.; Zysman-Colman, E. Advanced Materials 2017, 29, 1605444.

(26) Ziessel, R.; Retailleau, P.; Elliott, K. J.; Harriman, A. Chemistry - A European Journal 2009, 15, 10369-10374.

(27) Bureš, F. RSC Adv. 2014, 4, 58826-58851.

(28) grimme-lab/crest https://github.com/grimme-lab/crest (accessed Jun 3, 2020).

(29) Grimme, S. J. Chem. Theory Comput. 2019, 15, 2847-2862.

(30) Pracht, P.; Bohle, F.; Grimme, S. Phys. Chem. Chem. Phys. 2020, 22, 7169-7192.

(31) Pracht, P.; Caldeweyher, E.; Ehlert, S.; Grimme, S. ChemRxiv 2019.

(32) grimme-lab/xtb https://github.com/grimme-lab/xtb (accessed Jun 3, 2020).

(33) Grimme, S.; Bannwarth, C.; Shushkov, P. J. Chem. Theory Comput. 2017, 13, 19892009.

(34) Bannwarth, C.; Ehlert, S.; Grimme, S. J. Chem. Theory Comput. 2019, 15, 1652-1671.

(35) Neese, F. Wiley Interdisciplinary Reviews: Computational Molecular Science 2018, 8, e1327.

(36) Neese, F. WIREs Computational Molecular Science 2012, 2, 73-78.

(37) Becke, A. D. Phys. Rev. A 1988, 38, 3098-3100.

(38) Lee, C.; Yang, W.; Parr, R. G. Phys. Rev. B 1988, 37, 785-789.

(39) Becke, A. D. J. Chem. Phys. 1993, 98, 5648-5652.

(40) Dunning, T. H. J. Chem. Phys. 1989, 90, 1007-1023.

(41) Casanova-Páez, M.; Dardis, M. B.; Goerigk, L. J. Chem. Theory Comput. 2019, 15, 4735-4744.

(42) Weigend, F.; Ahlrichs, R. Phys. Chem. Chem. Phys. 2005, 7, 3297-3305.

(43) Guo, Y.; Sivalingam, K.; Valeev, E. F.; Neese, F. J. Chem. Phys. 2016, 144, 094111.

(44) Nielsen, E. S.; Jo/rgensen, P.; Oddershede, J. J. Chem. Phys. 1980, 73, 6238-6246.

(45) Sauer, S. P. A. J. Phys. B: At. Mol. Opt. Phys. 1997, 30, 3773-3780.

(46) Eriksen, J. J.; Sauer, S. P. A.; Mikkelsen, K. V.; Jensen, H. J. A.; Kongsted, J. Journal of Computational Chemistry 2012, 33, 2012-2022.

(47) Schirmer, J. Phys. Rev. A 1982, 26, 2395-2416.

(48) Trofimov, A. B.; Schirmer, J. J. Phys. B: At. Mol. Opt. Phys. 1995, 28, 2299-2324.

(49) Starcke, J. H.; Wormit, M.; Dreuw, A. J. Chem. Phys. 2009, 130, 024104.

(50) Wormit, M.; Rehn, D. R.; Harbach, P. H. P.; Wenzel, J.; Krauter, C. M.; Epifanovsky, E.; Dreuw, A. Molecular Physics 2014, 112, 774-784.

(51) ROWE, D. J. Rev. Mod. Phys. 1968, 40, 153-166.

(52) Emrich, K. Nuclear Physics A 1981, 351, 379-396.

(53) Geertsen, J.; Rittby, M.; Bartlett, R. J. Chemical Physics Letters 1989, 164, 57-62.

(54) Stanton, J. F.; Bartlett, R. J. J. Chem. Phys. 1993, 98, 7029-7039.

(55) Krylov, A. I. Annu. Rev. Phys. Chem. 2008, 59, 433-462.

(56) Landau, A.; Khistyaev, K.; Dolgikh, S.; Krylov, A. I. J. Chem. Phys. 2010, 132, 014109.

(57) Sosa, C.; Geertsen, J.; Trucks, G. W.; Bartlett, R. J.; Franz, J. A. Chemical Physics Letters 1989, 159, 148-154.

(58) Taube, A. G.; Bartlett, R. J. Collect. Czech. Chem. Commun. 2005, 70, 837-850.

(59) Taube, A. G.; Bartlett, R. J. J. Chem. Phys. 2008, 128, 164101.

(60) Krylov, A. I. Chemical Physics Letters 2001, 350, 522-530.

(61) Zhang, X.; Herbert, J. M. J. Chem. Phys. 2015, 143, 234107.

(62) Perdew, J. P.; Burke, K.; Ernzerhof, M. Phys. Rev. Lett. 1996, 77, 3865-3868.

(63) Perdew, J. P.; Ernzerhof, M.; Burke, K. J. Chem. Phys. 1996, 105, 9982-9985.

(64) Adamo, C.; Barone, V. J. Chem. Phys. 1999, 110, 6158-6170.

(65) Bernard, Y. A.; Shao, Y.; Krylov, A. I. The Journal of Chemical Physics 2012, 136, 204103.

(66) Shao, Y.; Gan, Z.; Epifanovsky, E.; Gilbert, A. T. B.; Wormit, M.; Kussmann, J.; Lange, A. W.; Behn, A.; Deng, J.; Feng, X.; et al. Molecular Physics 2015, 113, 184-215. 
(67) Baiardi, A.; Bloino, J.; Barone, V. J. Chem. Theory Comput. 2013, 9, 4097-4115.

(68) Grimme, S.; Neese, F. J. Chem. Phys. 2007, 127, 154116.

(69) Goerigk, L.; Moellmann, J.; Grimme, S. Physical Chemistry Chemical Physics 2009, 11, 4611-4620.

(70) Goerigk, L.; Grimme, S. J. Chem. Theory Comput. 2011, 7, 3272-3277.

(71) Schwabe, T.; Goerigk, L. J. Chem. Theory Comput. 2017, 13, 4307-4323.

(72) Shaw, J. T.; Prem, S. Journal of Heterocyclic Chemistry 1977, 14, 671-672.

(73) Pratap, R.; Roy, A. D.; Kushwaha, S. P.; Goel, A.; Roy, R.; Ram, V. J. Tetrahedron Letters 2007, 48, 5845-5849.

(74) Matsuda, Y.; Gotou, H.; Katou, K.; Matsumoto, H. Chemical \& Pharmaceutical Bulletin 1989, 37, 1188-1191.

(75) Shaw, J. T.; Coffindaffer, T. W.; Stimmel, J. B.; Lindley, P. M. Journal of Heterocyclic Chemistry 1982, 19, 357-361.

(76) Shaw, J. T.; Starkey, K. D.; Pelliccione, D. J.; Barnhart, S. L. Journal of Heterocyclic Chemistry 1983, 20, 1095-1097.

(77) Matsuo, M.; Awaya, H.; Maseda, C.; Tominaga, Y.; Natsuki, R.; Matsuda, Y.; Kobayashi, G. Chemical \& Pharmaceutical Bulletin 1974, 22, 2765-2766.

(78) Rossman, M. A.; Leonard, N. J.; Urano, S.; LeBreton, P. R. J. Am. Chem. Soc. 1985, 107, 3884-3890.

(79) Kanamori, K.; Roberts, J. D.; Rossman, M. A.; Leonard, N. J. Heteroatom Chemistry $1992,3,19-23$.

(80) Ceder, O.; Vernmark, K. Acta. Chem. Scand. B 1977, 31, 235-238.

(81) Boutique, J. P.; Verbist, J. J.; Fripiat, J. G.; Delhalle, J.; Pfister-Guillouzo, G.; Ashwell, G. J. J. Am. Chem. Soc. 1984, 106, 4374-4378.

(82) Watanabe, H.; Hirose, M.; Tanaka, K.; Tanaka, K.; Chujo, Y. Polym. Chem. 2016, 7 , 3674-3680.

(83) Yeo, H.; Hirose, M.; Tanaka, K.; Chujo, Y. Polym J 2014, 46, 688-693.

(84) Watanabe, H.; Hirose, M.; Tanaka, K.; Chujo, Y. Chem. Commun. 2017, 53, 5036-5039.

(85) Watanabe, H.; Kawano, Y.; Tanaka, K.; Chujo, Y. Asian Journal of Organic Chemistry 2020, 9, 259-266.

(86) Watanabe, H.; Tanaka, K.; Chujo, Y. J. Org. Chem. 2019, 84, 2768-2778.

(87) Winter, R. A. E.; Villani, T. J. 3886157, May 27, 1975.

(88) In Category 2, Hetarenes and Related Ring Systems; Thieme Verlag, 2004.

(89) Liu, Y.; Li, C.; Ren, Z.; Yan, S.; Bryce, M. R. Nat Rev Mater 2018, 3, 1-20.

(90) Kim, M.; Jeon, S. K.; Hwang, S.-H.; Lee, J. Y. Advanced Materials 2015, 27, 25152520.

(91) Lee, J.-H.; Chen, C.-H.; Lee, P.-H.; Lin, H.-Y.; Leung, M.; Chiu, T.-L.; Lin, C.-F. J. Mater. Chem. C 2019, 7, 5874-5888.

(92) Volz, D. JPE 2016, 6, 020901.

(93) Yang, Z.; Mao, Z.; Xie, Z.; Zhang, Y.; Liu, S.; Zhao, J.; Xu, J.; Chi, Z.; Aldred, M. P. Chem. Soc. Rev. 2017, 46, 915-1016.

(94) Data, P.; Takeda, Y. Chemistry - An Asian Journal 2019, 14, 1613-1636.

(95) Konidena, R. K.; Lee, J. Y. The Chemical Record 2019, 19, 1499-1517.

(96) Im, Y.; Kim, M.; Cho, Y. J.; Seo, J.-A.; Yook, K. S.; Lee, J. Y. Chem. Mater. 2017, 29, 1946-1963.

(97) Bergmann, L.; Zink, D. M.; Bräse, S.; Baumann, T.; Volz, D. Top Curr Chem (Z) 2016, 374, 22.

(98) Murata, I.; Yamamoto, K.; Morioka, M.; Tamura, M.; Hirotsu, T. Tetrahedron Letters 1975, 16, 2287-2288.

(99) Cunningham, R. P.; Farquhar, D.; Gibson, W. K.; Leaver, D. J. Chem. Soc. C 1969, No. 2, 239-243. 
(100) Farquhar, D.; Gough, T. T.; Leaver, D. J. Chem. Soc., Perkin Trans. 1 1976, No. 3 , $341-355$.

(101) Gibson, W. K.; Leaver, D. Chem. Commun. (London) 1965, No. 1, 11-11.

(102) Mamada, M.; Inada, K.; Komino, T.; Potscavage, W. J.; Nakanotani, H.; Adachi, C. ACS Cent. Sci. 2017, 3, 769-777.

(103) Kwon, J. E.; Park, S. Y. Advanced Materials 2011, 23, 3615-3642.

(104) Wu, K.; Zhang, T.; Wang, Z.; Wang, L.; Zhan, L.; Gong, S.; Zhong, C.; Lu, Z.-H.; Zhang, S.; Yang, C. J. Am. Chem. Soc. 2018, 140, 8877-8886.

(105) Long, Y.; Mamada, M.; Li, C.; dos Santos, P. L.; Colella, M.; Danos, A.; Adachi, C.; Monkman, A. J. Phys. Chem. Lett. 2020, 11, 3305-3312.

(106) Cao, Y.; Eng, J.; Penfold, T. J. J. Phys. Chem. A 2019, 123, 2640-2649.

(107) Padalkar, V. S.; Seki, S. Chem. Soc. Rev. 2015, 45, 169-202.

(108) Li, B.; Zhou, Q.; Sun, C.; Cao, B.; Li, Y.; Han, J.; Yin, H.; Shi, Y. Spectrochimica Acta Part A: Molecular and Biomolecular Spectroscopy 2020, 239, 118386.

(109) Jiang, G.; Li, F.; Fan, J.; Song, Y.; Wang, C.-K.; Lin, L. Journal of Materials Chemistry C 2020, 8, 98-108.

(110) Zhang, N.; Zhang, T.; Wen, L.; Wang, L.; Yan, J.; Zheng, K. Physical Chemistry Chemical Physics 2020, 22, 1409-1415.

(111) Wei, Q.; Fei, N.; Islam, A.; Lei, T.; Hong, L.; Peng, R.; Fan, X.; Chen, L.; Gao, P.; Ge, Z. Advanced Optical Materials 2018, 6, 1800512.

(112) Uoyama, H.; Goushi, K.; Shizu, K.; Nomura, H.; Adachi, C. Nature 2012, 492, 234 238.

(113) Nakanotani, H.; Higuchi, T.; Furukawa, T.; Masui, K.; Morimoto, K.; Numata, M.; Tanaka, H.; Sagara, Y.; Yasuda, T.; Adachi, C. Nat Commun 2014, 5, 1-7.

(114) Casida, M. E.; Huix-Rotllant, M. Annual Review of Physical Chemistry 2012, 63, 287323.

(115) Kimber, P.; Plasser, F. 2020.

(116) de Silva, P.; Kim, C. A.; Zhu, T.; Van Voorhis, T. Chem. Mater. 2019, 31, 6995-7006.

(117) Liang, X.; Tu, Z.-L.; Zheng, Y.-X. Chemistry - A European Journal 2019, 25, 56235642.

(118) Kollmar, H.; Staemmler, V. Theoret. Chim. Acta 1978, 48, 223-239.

(119) In Electron Spin Resonance Spectroscopy of Organic Radicals; John Wiley \& Sons, Ltd, 2004; pp 49-82.

(120) Borden, W. T. J. Am. Chem. Soc. 1975, 97, 5968-5970.

(121) Bally, T.; Borden, W. T. In Reviews in Computational Chemistry; John Wiley \& Sons, Ltd, 2007; pp 1-97.

(122) Goerigk, L.; Casanova-Paéz, M. Aust. J. Chem. 2020.

(123) Casanova, D.; I. Krylov, A. Physical Chemistry Chemical Physics 2020.

(124) Hrovat, D. A.; Borden, W. T. Journal of Molecular Structure: THEOCHEM 1997, 398-399, 211-220.

(125) Gómez-Bombarelli, R.; Aguilera-Iparraguirre, J.; Hirzel, T. D.; Duvenaud, D.; Maclaurin, D.; Blood-Forsythe, M. A.; Chae, H. S.; Einzinger, M.; Ha, D.-G.; Wu, T.; et al. Nature Materials 2016, 15, 1120-1127.

(126) Shu, Y.; Levine, B. G. J. Chem. Phys. 2015, 142, 104104.

(127) Penfold, T. J. J. Phys. Chem. C 2015, 119, 13535-13544.

(128) Tsuchiya, Y.; Tsuji, K.; Inada, K.; Bencheikh, F.; Geng, Y.; Kwak, H. S.; Mustard, T. J. L.; Halls, M. D.; Nakanotani, H.; Adachi, C. Front. Chem. 2020, 8.

(129) Hirata, S.; Sakai, Y.; Masui, K.; Tanaka, H.; Lee, S. Y.; Nomura, H.; Nakamura, N.; Yasumatsu, M.; Nakanotani, H.; Zhang, Q.; et al. Nature Materials 2015, 14, 330-336.

(130) Hatakeyama, T.; Shiren, K.; Nakajima, K.; Nomura, S.; Nakatsuka, S.; Kinoshita, K.; Ni, J.; Ono, Y.; Ikuta, T. Advanced Materials 2016, 28, 2777-2781. 
(131) Pershin, A.; Hall, D.; Lemaur, V.; Sancho-Garcia, J.-C.; Muccioli, L.; ZysmanColman, E.; Beljonne, D.; Olivier, Y. Nat Commun 2019, 10, 1-5.

(132) Zhang, Y.; Zhang, D.; Wei, J.; Hong, X.; Lu, Y.; Hu, D.; Li, G.; Liu, Z.; Chen, Y.; Duan, L. Angewandte Chemie International Edition Just Accepted.

(133) Liang, X.; Yan, Z.-P.; Han, H.-B.; Wu, Z.-G.; Zheng, Y.-X.; Meng, H.; Zuo, J.-L.; Huang, W. Angewandte Chemie International Edition 2018, 57, 11316-11320.

(134) Oda, S.; Kawakami, B.; Kawasumi, R.; Okita, R.; Hatakeyama, T. Org. Lett. 2019, 21, 9311-9314.

(135) Yuan, Y.; Tang, X.; Du, X.-Y.; Hu, Y.; Yu, Y.-J.; Jiang, Z.-Q.; Liao, L.-S.; Lee, S.-T. Advanced Optical Materials 2019, 7, 1801536.

(136) Hall, D.; Suresh, S. M.; Santos, P. L. dos; Duda, E.; Bagnich, S.; Pershin, A.; Rajamalli, P.; Cordes, D. B.; Slawin, A. M. Z.; Beljonne, D.; et al. Advanced Optical Materials 2020, 8, 1901627.

(137) Zhang, Y.; Zhang, D.; Wei, J.; Liu, Z.; Lu, Y.; Duan, L. Angewandte Chemie International Edition 2019, 58, 16912-16917.

(138) Nakanotani, H.; Furukawa, T.; Hosokai, T.; Hatakeyama, T.; Adachi, C. Advanced Optical Materials 2017, 5, 1700051.

(139) Lee, J.; Shizu, K.; Tanaka, H.; Nomura, H.; Yasuda, T.; Adachi, C. J. Mater. Chem. C 2013, 1, 4599-4604.

(140) Tao, Y.; Yuan, K.; Chen, T.; Xu, P.; Li, H.; Chen, R.; Zheng, C.; Zhang, L.; Huang, W. Advanced Materials 2014, 26, 7931-7958.

(141) Samuel, I. D. W.; Turnbull, G. A. Chem. Rev. 2007, 107, 1272-1295.

(142) Oyama, Y.; Mamada, M.; Shukla, A.; Moore, E. G.; Lo, S.-C.; Namdas, E. B.; Adachi, C. ACS Materials Lett. 2020, 2, 161-167.

(143) Chen, X.-K.; Kim, D.; Brédas, J.-L. Acc. Chem. Res. 2018, 51, 2215-2224.

(144) Peng, Q.; Fan, D.; Duan, R.; Yi, Y.; Niu, Y.; Wang, D.; Shuai, Z. J. Phys. Chem. C 2017, 121, 13448-13456.

(145) Li, J.; Nakagawa, T.; MacDonald, J.; Zhang, Q.; Nomura, H.; Miyazaki, H.; Adachi, C. Advanced Materials 2013, 25, 3319-3323.

(146) Li, J.; Nomura, H.; Miyazaki, H.; Adachi, C. Chem. Commun. 2014, 50, 6174-6176.

(147) Li, J.; Zhang, Q.; Nomura, H.; Miyazaki, H.; Adachi, C. Appl. Phys. Lett. 2014, 105, 013301.

(148) Northey, T.; Penfold, T. J. Organic Electronics 2018, 59, 45-48.

(149) Lin, L.; Fan, J.; Cai, L.; Wang, C.-K. Molecular Physics 2018, 116, 19-28.

(150) Penfold, T. J.; Gindensperger, E.; Daniel, C.; Marian, C. M. Chem. Rev. 2018, 118, $6975-7025$.

(151) Gibson, J.; Penfold, T. J. Phys. Chem. Chem. Phys. 2017, 19, 8428-8434.

(152) Gibson, J.; Monkman, A. P.; Penfold, T. J. ChemPhysChem 2016, 17, 2956-2961.

(153) Etherington, M. K.; Gibson, J.; Higginbotham, H. F.; Penfold, T. J.; Monkman, A. P. Nat Commun 2016, 7, 1-7.

(154) Marian, C. M. J. Phys. Chem. C 2016, 120, 3715-3721.

(155) Head-Gordon, M.; Rico, R. J.; Oumi, M.; Lee, T. J. Chemical Physics Letters 1994, 219, 21-29.

(156) Head-Gordon, M.; Maurice, D.; Oumi, M. Chemical Physics Letters 1995, 246, 114 121.

(157) Kats, D.; Schütz, M. J. Chem. Phys. 2009, 131, 124117.

(158) Kats, D.; Korona, T.; Schütz, M. J. Chem. Phys. 2006, 125, 104106.

(159) Ledermüller, K.; Schütz, M. J. Chem. Phys. 2014, 140, 164113.

(160) Freundorfer, K.; Kats, D.; Korona, T.; Schütz, M. J. Chem. Phys. 2010, 133, 244110.

(161) Kats, D.; Schütz, M. Zeitschrift für Physikalische Chemie 2010, 224, 601-616.

(162) Schütz, M. J. Chem. Phys. 2015, 142, 214103. 
(163) Kats, D.; Korona, T.; Schütz, M. J. Chem. Phys. 2007, 127, 064107.

(164) Ehrmaier, J.; Huang, X.; Rabe, E. J.; Corp, K. L.; Schlenker, C. W.; Sobolewski, A. L.; Domcke, W. J. Phys. Chem. A 2020.

(165) Ceder, Olof; Widing, Per-Olof; Vernmark, Karin. Acta Chem. Scand. 1976, 30b, 466468. 